\title{
INFLUÊNCIA DA DEPENDÊNCIA DE PARÂMETROS DO SOLO COM A FREQUÊNCIA NA MODELAGEM DE LINHAS AÉREAS DE TRANSMISSÃO: CASO DE CONDUTOR ÚNICO
}

\author{
J. B. Gertrudes* \\ jbosco@dsce.fee.unicamp.br \\ M. C. Tavares* \\ cristina@dsce.fee.unicamp.br \\ C. Portela ${ }^{\dagger}$ \\ portelaceism.com.br \\ *Universidade Estadual de Campinas \\ DSCE/FEEC/UNICAMP \\ CEP 13083-852, Campinas/SP, Brasil \\ ${ }^{\dagger}$ Universidade Federal do Rio de Janeiro - COPPE/UFRJ \\ Rua Eng. César Grillo 249, \\ CEP 22640-150 Rio de Janeiro, RJ, Brasil
}

\begin{abstract}
Influence of earth's conductivity and permittivity frequency dependence on overhead transmission lines: analysis for the one - conductor case

In this paper the influence of earth's conductivity and permittivity frequency dependence are evaluated when calculating transversal and longitudinal transmission lines' parameters. The aim is to compare results calculated from the complex plane method and Carson's modified expressions on longitudinal parameters and also between Carson's modified potential-coefficient correction factors and results from approximations methods for transversal admittance parameters.
\end{abstract}

The importance of properly considering the frequency-dependent soil model is presented for oneconductor case, in order to compare the longitudinal and transversal parameters considering the earth's conductivity and permittivity frequency dependence soil model in relation to the common soil representation with a constant

Artigo submetido em 03/12/2009 (Id.: 01089)

Revisado em 18/02/2010, 12/07/2010, 14/09/2010.

Aceito em 03/11/2010 sob recomendação do Editor Associado Prof. Antonio Carlos Zambroni de Souza conductivity and a permittivity that can be neglected assuming a low frequency approximation.

KEYWORDS: Soil model, Line parameters,Electromagnetic transients.

\section{RESUMO}

Este trabalho enquadra-se na linha de pesquisa que tem como objetivo a busca de modelos mais precisos para cálculo de parâmetros de linhas aéreas de transmissão na faixa de frequência 0 a $2 \mathrm{MHz}$. Esta faixa de frequência cobre a maioria das perturbações eletromagnéticas nos sistemas elétricos (manobras, ocorrências de faltas, rejeições de carga, descargas atmosféricas, perturbações harmônicas). Entre $1 \mathrm{~Hz}$ e $2 \mathrm{MHz}$ o solo pode ter a condutividade elétrica da mesma ordem de grandeza que o produto entre a constante dielétrica e a frequência angular do sinal incidente no solo, em função da dependência desses parâmetros com a frequência. Consequentemente, as suposições de baixa frequência tradicionalmente adotadas - condutividade do solo constante e permissividade desprezível - podem levar a modelos que não representam de forma adequada a resposta 
da linha no caso de transitórios rápidos (com espectro de frequência acima de $1 \mathrm{kHz}$ ).

É apresentado um estudo dos parâmetros de uma linha formada por um único condutor acima de solo condutor imperfeito, - onde a impedância e a admitância de retorno pelo solo são avaliadas através de métodos tradicionais de cálculo e através de integração numérica das formulações de Carson modificadas para inclusão do modelo de solo com parâmetros dependentes da frequência.

PALAVRAS-CHAVE: Parâmetros do solo, Linhas de transmissão, Transitórios eletromagnéticos.

\section{INTRODUÇÃO}

O problema da propagação de campos eletromagnéticos ao longo de uma linha de transmissão composta por um único condutor acima do solo (considerado um meio com perdas) foi inicialmente estudado por Carson (Carson, 1926). As formulações de Carson foram derivadas com algumas suposições ou limitações: (i) solo como meio linear isotrópico e homogêneo com permeabilidade magnética relativa unitária; (ii) propagação de ondas na velocidade da luz e sem atenuação na direção axial ao eixo do condutor; (iii) condição de baixa frequência (condutividade do solo constante e permissividade desprezível). Estas restrições implicam em soluções com razoável precisão até $1 \mathrm{MHz}$, que dependem da configuração de condutores na torre, características do solo, dimensões do circuito e comprimento de onda.

Seguindo a mesma linha de pesquisa, foram publicadas trabalhos com extensões nas formulações de Carson em 1931 e 1934. No primeiro trabalho (Wise, 1931) foram apresentadas correções nas formulações de Carson para inclusão do efeito da permissividade do solo, ou seja, resolvendo o problema da condição de baixa frequência. Até então as formulações eram relativas a impedância longitudinal de retorno pelo solo. No segundo trabalho (Wise, 1934) foram derivados os fatores de correção para admitância transversal com base no conceito de potenciais de Hertz, considerando o solo como plano condutor imperfeito.

A determinação das correções tanto para a impedância longitudinal quanto para a admitância transversal era na época relativamente trabalhosa, já que dependiam de integrações infinitas com argumentos complexos. A dificuldade de implementação computacional e avaliação das integrais infinitas tornou-se, na época, a principal motivação para diversas pesquisas subsequentes.
Posteriormente, foi apresentado um estudo propondo termos de correção na matriz de coeficientes de potencial e, consequentemente, na admitância transversal para um sistema com n-condutores. Os fatores de correção foram calculados a partir de aproximações sucessivas baseadas no método das imagens (Arismunandar, 1963).

Em 1981, surge um dos primeiros trabalhos com resultados numéricos calculados via integração numérica das formulações de Carson/Wise com correções simultaneamente na impedância longitudinal e admitância transversal (Nakagawa, 1981). No referido artigo ficou evidente que a desconsideração dos termos de correção na admitância resultaria em constantes de atenuação com erros consideráveis em relação aos modelos que desprezam a influência do solo na admitância transversal.

Com relação ao cálculo da impedância longitudinal convém destacar o método do plano complexo (Deri, 1981) que introduziu o conceito de distância equivalente de retorno para inclusão do efeito do solo nos cálculos, seguindo a mesma linha de pesquisa de busca por soluções para a integral infinita de Carson. Trata-se de aproximações assintóticas de primeira ordem das integrais de Carson cuja precisão depende da frequência, raio dos condutores, configuração dos condutores na torre, frequência do sinal e dos parâmetros do solo. O método é amplamente utilizado, mas os erros assintóticos devem ser avaliados para cada caso particular.

Nos trabalhos anteriormente citados não se considera a dependência dos parâmetros do solo com a frequência no cálculo da impedância longitudinal e da admitância transversal de retorno pelo solo.

Em 1997 foram apresentados estudos e procedimentos para modelagem matemática e de medição em campo dos parâmetros do solo no domínio da frequência, na faixa de 0 a $2 \mathrm{MHz}$ (Portela, 1997). A referida modelagem satisfaz as condições de coerência física em relação à propagação dos campos eletromagnéticos tendo em conta a dependência dos parâmetros do solo com a frequência. Os modelos foram testados com resultados de medições em campo analisados em 68 amostras de solo na Região Amazônica e posteriormente em 10 amostras de solo na região de Cachoeira Paulista/SP. O modelo desenvolvido representa a dependência da condutividade $\left(\sigma_{g}\right)$ e do produto entre a constante dielétrica $\left(\epsilon_{g}\right)$ e a frequência angular do sinal incidente no solo $(\omega)$ através de ajuste de parâmetros a partir das medições de campo. O produto $\omega \epsilon_{g}$ está relacionado à densidade de correntes de deslocamentos induzidas no solo que normalmente são desconsideradas nos cálculos assumindo a condição para baixas freqüências em que $\omega \epsilon_{g}<<\sigma_{g}$. 
Trabalhos subsequentes com base no plano complexo modificado (Portela et alii, 2003), (Portela et alii, 2006) concluem que a dependência dos parâmetros do solo com a frequência no cálculo da impedância longitudinal tem influência significativa nos parâmetros de propagação e resposta da linha na faixa de frequência de $1 \mathrm{~Hz}$ a $1 \mathrm{MHz}$, ainda com a admitância transversal calculada na condição de solo ideal.

Este trabalho vem na sequência das pesquisas até então realizadas, tendo como principal motivação verificar a influência da dependência de $\sigma_{g}$ e $\omega \epsilon_{g}$ com a frequência tanto no cálculo da impedância longitudinal quanto no cálculo da admitância transversal por unidade de comprimento. São feitas análises e comparações para o caso de um único condutor acima do solo com parâmetros dependentes da frequência. A impedância longitudinal e a admitância transversal de retorno pelo solo são avaliadas através de métodos tradicionais de cálculo e através de integração numérica das formulações de Carson, modificadas para inclusão do modelo de solo que considera a dependência $\sigma_{g}$ e $\omega \epsilon_{g}$ com a frequência.

\section{DINÂMICA DA PROPAGAÇÃO DE ONDAS ELETROMAGNÉTICAS EM LINHAS DE TRANSMISSÃO: HIPÓTESES BÁSICAS NO CÁLCULO TRADICIONAL DE PARÂMETROS}

A dinâmica de propagação de ondas eletromagnéticas para o caso de um único condutor acima do solo, considerando o modelo a parâmetros distribuídos e representação complexa da corrente e tensão senoidal, é determinada pelas seguintes equações diferenciais que relacionam a tensão transversal $(V)$ entre um ponto na superfície do condutor e o solo e a corrente longitudinal ( $I$ ), que flui dentro do condutor, com a impedância longitudinal e admitância transversal por unidade de comprimento do condutor:

$$
\begin{gathered}
\frac{d^{2} V}{d x^{2}}=Z Y V \quad \frac{d^{2} I}{d x^{2}}=Z Y I \\
Y=G+i \omega C
\end{gathered}
$$

onde $x$ é a distância ao longo da linha assumindo a carga como referência; $Z$ é a impedância longitudinal por unidade de comprimento; $Y$ é a admitância transversal por unidade de comprimento; $\omega$ é a frequência angular do sinal; $i$ é o número imaginário puro.

A solução do sistema de equações em (1) depende das condições de contorno no terminal gerador e na carga e das suposições no cálculo da impedância longitudinal e admitância transversal da linha de transmissão.

As equações de propagação em (1) são válidas considerando as suposições relativas a geometria da linha descritas a seguir.

As simplificações de geometria utilizadas para o cálculo de $\mathrm{Z}$ e Y consistem em: considerar a superfície do solo plano; considerar o cabo paralelo ao solo; considerar a distância condutor-solo muito maior do que o raio do mesmo. As mudanças de temperatura devido a condições de carregamento da linha e meteorológicas (vento, chuva, gelo) têm influência no posicionamento do condutor ao longo de todo o trecho da linha. Portanto, o posicionamento do condutor na torre é dinâmico e de difícil modelagem. Normalmente considera-se o condutor à uma altura média $\left(h_{m}\right)$ em relação ao solo perfeitamente plano. As variações de altura decorrentes de variações de temperatura, de carregamento e do relevo são desta forma aproximadas. Além disso, o solo não é perfeitamente plano resultando em variação de altura do condutor em relação ao solo ao longo do vão e, consequentemente, ao longo de toda a extensão da linha. Para vãos muito longos e locais íngremes o uso da altura média acarretará em erros maiores e uma representação mais acurada da altura dos condutores deve ser empregada. Na modelagem efetuada neste trabalho, os cálculos foram feitos considerando a temperatura do cabo de $75^{\circ} \mathrm{C}$.

Algumas suposições de propagação quase-estacionárias são tradicionalmente assumidas no cálculo da impedância longitudinal e admitância transversal, por exemplo: considerar o tempo de propagação nulo entre condutor e solo; relacionar, no mesmo instante, o campo elétrico e o magnético estritamente às correntes e às cargas no plano perpendicular ao eixo da linha; simplificações de geometria nas condições de contorno nas superfícies condutor/ar e ar/solo. Assumir o solo estritamente homogêneo ou com camadas homogêneas separadas por planos perfeitamente horizontais; assumir as mesmas características do solo ao longo de toda extensão da linha; considerar linear a relação $\mathrm{BxH}$ nos materiais de que são constituídos o cabo condutor (alumínio com alma de aço), com permeabilidade magnética, $\mu$, aproximadamente igual à do vácuo, supondo que a corrente na alma de aço é nula. Os efeitos no campo eletromagnético das estruturas, sistema de aterramento e eventuais cabos contrapesos são desprezados.

Quando as simplificações mencionadas são potencialmente importantes elas devem ser incorporadas na modelagem de cálculo dos parâmetros. 


\subsection{Impedância longitudinal por unidade de comprimento: Métodos de cálculo aproximado}

Considerando as suposições descritas acima, a impedância longitudinal, $Z$, é obtida de forma aproximada somando-se três parcelas distintas, cada uma delas com significante contribuição:

$$
Z=Z_{\text {int }}+Z_{\text {ext }}+Z_{g}
$$

- A impedância longitudinal interna, $Z_{\text {int }}$, por unidade de comprimento, associada à propagação de campo eletromagnético no interior do condutor (efeito pelicular), supondo o solo como um plano condutor perfeito. Esta impedância pode ser calculada através de fórmulas baseadas nas equações de Bessel.

- A impedância longitudinal externa, $Z_{e x t}$, por unidade de comprimento, associada à propagação de campo eletromagnético no ar. Esta parcela é obtida com erros desprezíveis ou aceitáveis até cerca de $1 \mathrm{MHz}$, assumindo condutores ideais e o solo plano condutor perfeito e campo eletromagnético quase estacionário.

- A impedância longitudinal de retorno pelo solo, $Z_{g}$, por unidade de comprimento, devido à propagação do campo eletromagnético no solo, quando este não é considerado um meio com perdas. Para solos homogêneos, exceto para o caso em que a permeabilidade magnética do solo é diferente da do vácuo - caso em que não se pode aplicar a formulação de Carson original - a impedância de retorno pelo solo pode ser calculada com erro aceitável até frequências da ordem de $1 \mathrm{MHz}$ através de formulações de Carson modificadas. As modificações referidas consistem na substituição de $\sigma_{g}$ por $\sigma_{g}+\mathrm{i} \omega \epsilon_{g}$. Mais precisamente, nas formulações de Carson ou equivalentes, o coeficiente de propagação $\gamma_{g}=\sqrt{i \mu_{g} \omega\left(\sigma_{g}+i \omega \varepsilon_{g}\right)}$ deve ser utilizado ao invés de $\gamma_{g}=\sqrt{i \mu_{g} \omega \sigma_{g}}$. Esta última consideração é válida somente para campos quase estacionários, ou seja, em que a permissividade elétrica do solo possa ser desprezada.

Os resultados de trabalhos anteriores (Tavares et alii, 2003), (Portela et alii, 2006) mostram erros consideráveis no cálculo da impedância longitudinal por unidade de comprimento quando comparados com os modelos quase estacionários.

Nas mesmas condições de aplicabilidade, a impedância longitudinal total (externa + retorno pelo solo) por unidade de comprimento pode ser calculada com erros aceitáveis utilizando o método do plano complexo modificado. O método consiste em aproximações assintóticas das integrais de Carson (também substituindo $\sigma_{g}$ por $\sigma_{g}+\mathrm{i} \omega \epsilon_{g}$, seguindo a mesma linha de raciocínio). O efeito do solo é incluído diretamente na impedância longitudinal externa por unidade de comprimento utilizando-se o método das imagens, isto é, considerando o solo como um plano condutor ideal a uma profundidade complexa $d^{\prime}=1 / \sqrt{\left(\sigma_{g}+i \omega \varepsilon_{g}\right) i \mu_{g} \omega}$ abaixo da superfície do solo real. Esta suposição é tratada como uma correção nas formulações aproximadas Dubanton/Deri pela substituição da profundidade complexa presente na referida publicação, dada por $d=1 / \sqrt{i \omega \mu_{g} \sigma_{g}}$ pela profundidade complexa dada pela expressão acima. $\mathrm{O}$ método do plano complexo modificado (Deri-M) tem sido utilizado para inclusão do efeito do solo nos parâmetros longitudinais em trabalhos anteriores e permite o cálculo das parcelas $Z_{e x t}$ e $Z_{g}$ através de uma expressão de rápida implementação computacional definida pela equação (7) .

O erro assintótico do método do plano complexo depende da frequência e da geometria da linha (relação entre a distância horizontal dos condutores e altura destes em relação ao solo) e sua utilização deve ser analisada para cada caso específico. Há casos em que o erro pode chegar a mais de $15 \%$ e comprometer a eficácia do método. O cálculo da contribuição do solo pode ser efetuado com maior grau de exatidão através das formulações de Carson por integração numérica.

A impedância interna dos condutores $\left(Z_{\text {int }}\right)$ varia com a frequência devido ao efeito pelicular e é obtida através do quociente entre campo elétrico longitudinal na superfície do cabo condutor e a corrente no interior do mesmo. Esta parcela depende essencialmente dos parâmetros elétricos do condutor $\left(\sigma_{c}\right.$ e $\left.\mu_{c c}\right)$ e das características físicas (raio interno e externo). A formulação matemática da impedância interna longitudinal por unidade de comprimento é conhecida desde 1918 (Dwicht, 1918):

$$
\begin{gathered}
Z_{\mathrm{int}}=\sqrt{\frac{i \omega \mu_{c}}{\sigma}} \frac{1}{2 \pi R_{1}} \frac{I_{0}\left(\rho_{1}\right) K_{1}\left(\rho_{0}\right)+K_{0}\left(\rho_{1}\right) I_{1}\left(\rho_{0}\right)}{I_{1}\left(\rho_{1}\right) K_{1}\left(\rho_{0}\right)-K_{1}\left(\rho_{1}\right) I_{1}\left(\rho_{0}\right)} \\
\rho_{0}=R_{0} \sqrt{i \omega \mu_{c} \sigma_{c}}=R_{0} \sqrt{\omega \mu_{c} \sigma_{c}} e^{i \frac{\pi}{4}} \\
\rho_{1}=R_{1} \sqrt{i \omega \mu_{c} \sigma_{c}}=R_{1} \sqrt{\omega \mu_{c} \sigma_{c}} e^{i \frac{\pi}{4}}
\end{gathered}
$$

onde:

$I_{0}, I_{1}, K_{0}, K_{1}$ - são as funções modificadas de Bessel de primeira espécie $(I)$ e segunda espécie $(K)$ e de ordem zero e um, respectivamente;

$\omega$ - frequência angular do sinal de excitação;

$\sigma_{c}$ - condutividade do condutor; 
$\mu_{c}$ - permeabilidade magnética do condutor;

$R_{0}$ - raio da alma de aço do condutor ou valor nulo para condutor sólido;

$R_{1}$ - raio externo do condutor;

A expressão da impedância externa é obtida pelo método das imagens (fluxo total por unidade de comprimento concatenado entre condutor e a superfície do solo - dividido pela corrente que flui no interior do condutor).

$$
Z_{\text {ext }}+Z_{g}(\text { Deri }-M)=i \frac{\omega \mu}{2 \pi} \ln \left(\frac{2\left(h+d^{\prime}\right)}{R_{1}}\right)
$$

onde:

$h$ - altura média do condutor em relação ao solo;

$d^{\prime}$ - distância complexa de retorno equivalente;

A impedância de retorno pelo solo pode ser calculada de forma aproximada pela expressão de Sunde (1968). Esta expressão foi deduzida considerando a parcela referente às correntes de deslocamento na formulação de Maxwell e é equivalente à expressão da Deri modificada (Deri-M):

$$
Z_{g}(S-M) \cong i \frac{\omega \mu}{\pi} \ln \left(\frac{1+\gamma_{g} h}{\gamma_{g} h}\right)
$$

\subsection{Formulações modificadas de Carson/Wise/Nakagawa (C/W/N-M) na forma integral para o cálculo da Impedância e admitância de retorno pelo solo}

$\mathrm{Na}$ década de 90 foram derivados a partir do vetor potencial de Hertz fatores de correção na forma integral para a impedância longitudinal, quando o solo não é considerado um plano condutor perfeito e com permeabilidade magnética diferente da do vácuo - uma das limitações das formulações de Carson/Wise (Nakagawa, 1991). Com o conceito de vetor potencial de Hertz a maioria das limitações das formulações de Carson foram solucionadas, mantendo apenas a condição da distância entre condutor e solo ser muito maior que o raio do mesmo. No referido artigo, os parâmetros do solo $\left(\sigma_{g}\right.$ e $\left.\omega \epsilon_{g}\right)$ foram considerados constantes, sendo proposto no presente trabalho a inclusão da sua dependência com a frequência.

Considerando a definição do potencial escalar, $V$, de um condutor acima do solo na condição de plano condutor imperfeito, dado por:

$$
V=-\operatorname{div} \vec{\varphi}
$$

onde $\varphi$ é vetor potencial de Hertz. O coeficiente de potencial $P$ do condutor é definido como $V=Q P$, onde $Q$ é a carga por unidade de comprimento no condutor em $\mathrm{C} / \mathrm{m}$. $\mathrm{O}$ vetor potencial de Hertz (ou vetor de Hertz) de um condutor infinitamente longo é obtido por meio de integração das componentes do potencial de um dipolo de corrente acima do solo considerado condutor imperfeito expressa por (Nakagawa, 1981):

$\varphi_{x}=\frac{i \omega \mu I}{2 \pi \gamma_{0}^{2}}\left(\ln \left(\frac{2 h}{R_{1}}\right)+2 \int_{0}^{\infty} \frac{e^{-2 h \xi}}{\xi+\frac{\mu}{\mu_{g}} a_{1-f d}} d \xi\right)$

$\varphi_{y}=0$

$$
\varphi_{z}=\frac{i \omega \mu I}{2 \pi \gamma_{0}^{2}}\left(2 \gamma_{0}\right)\left(\int_{0}^{\infty} \frac{\left(1-\tau_{f d^{2}}\right) e^{-2 h \xi}}{\left(\xi+\frac{\mu}{\mu_{g}} a_{1-f d}\right)\left(\xi+\frac{\mu_{g}}{\mu} \tau_{f d^{2}} a_{1-f d}\right)} d \xi\right)
$$

onde:

$\mu, \epsilon$ - permeabilidade magnética e permissividade elétrica do ar;

$\mu_{g}, \epsilon_{g}$ - permeabilidade magnética e permissividade elétrica do solo;

$\gamma_{0}=i \omega \sqrt{\mu \varepsilon}-$ coeficiente de propagação no ar;

$\gamma_{g}=\sqrt{i \omega \mu_{g}\left(\sigma_{g}+i \omega \varepsilon_{g}\right)}$ - coeficiente de propagação no solo;

$$
\begin{aligned}
a_{1-f d} & =\sqrt{\xi^{2}+\gamma_{g-f d^{2}-\gamma_{0}^{2}}} \\
\tau_{f d}{ }^{2} & =\frac{\gamma_{0}^{2}}{\gamma_{g-f d^{2}}}
\end{aligned}
$$

Respeitando as condições de aplicabilidade e limitações, as fórmulas derivadas das equações de Carson que consideram $\epsilon_{g}=0$ podem ser utilizadas para incorporação do efeito da dependência dos parâmetros do solo com a frequência, substituindo $\sigma_{g}$ por $\sigma_{g}+\mathrm{i} \omega \epsilon_{g}$, com maior ou menor grau de exatidão dependendo das suposições relativas à propagação de ondas. Os parâmetros do solo $\sigma_{g}+\mathrm{i} \omega \epsilon_{g}$ surgem naturalmente nas equações de Maxwell no domínio da frequência em formulação complexa. Neste trabalho foi utilizado o modelo tipo 3 (Portela, 1999) que leva em 
consideração a dependência dos parâmetros do solo com a frequência. Os detalhes de modelagem matemática e procedimentos de medição em campo foram publicados em diversos artigos anteriores e citados nas referências. O modelo físico representa a dependência de $\sigma_{g}+\mathrm{i} \omega \epsilon_{g}$ através de ajuste dos parâmetros $\left(K_{0}, K_{1}, \alpha_{1}\right)$ a partir das medições de campo:

$$
\sigma_{g}+i \omega \epsilon_{g}=K_{0}+K_{1} \omega^{\alpha_{1}}+i K_{1} \tan \left(\frac{\pi}{2} \alpha_{1}\right) \omega^{\alpha_{1}}
$$

Este modelo define os parâmetros do solo em toda a faixa de frequência em estudo (0 a $2 \mathrm{MHz}$ ). Portanto, nas formulações originalmente derivadas, deve-se substituir $\gamma_{g}$ pela expressão do coeficiente de propagação, resultando em formulações modificadas com os parâmetros do solo dependentes da frequência (indicado nas expressões pelo índice " $f d$ "):

$\gamma_{g-f d}=\sqrt{i \omega \mu_{g}\left[\left(K_{0}+K_{1} \omega^{\alpha_{1}}+i K_{1} \tan \left(\frac{\pi}{2} \alpha_{1}\right) \omega^{\alpha_{1}}\right)\right]}$

A impedância longitudinal por unidade de comprimento é obtida diferenciando cada componente do vetor potencial de Hertz acima (10.a-c) com respeito a cada direção e, em seguida, substituindo em (9) obtém-se a expressão da impedância externa total por unidade de comprimento e o termo de correção devido ao retorno pelo solo $(p)$, dados respectivamente por:

$$
\begin{gathered}
Z_{e x t}+Z_{g}(C / W / N-M)=i \omega \frac{\mu_{o}}{2 \pi}\left[\ln \left(\frac{2 h}{R_{1}}\right)+p(\omega, \xi)\right] \\
p(\omega, \xi)=2 \int_{0}^{\infty} \frac{e^{-2 h \xi}}{\xi+\frac{\mu_{0}}{\mu_{g}} a_{1-f d}} d \xi
\end{gathered}
$$

A admitância transversal com correção devido ao retorno pelo solo é obtida de forma análoga a partir do vetor potencial de Hertz. Das equaões de onda têm-se:

$$
-\frac{d I}{d x}=i \omega Q=Y V
$$

Portanto, diferenciando cada componente do vetor potencial de Hertz acima (10.a-c) com respeito a cada direção e substituindo em (9) e (16), respectivamente, obtém-se a expressão de admitância externa total por unidade de comprimento corrigida $\left(Y_{c}\right)$, o coeficiente de potencial $(P)$ e o termo de correção devido ao retorno pelo solo $(q)$, dados respectivamente por:

$$
\begin{aligned}
& Y_{c}(C / W / N-M)=i \omega P^{-1} \\
& P=\frac{1}{2 \pi \varepsilon}\left[\ln \left(\frac{2 h}{R_{1}}\right)+q(\omega, \xi)\right] \\
& q(\omega, \xi)=2 \int_{0}^{\infty} \frac{\left(\xi+\frac{\mu_{g}}{\mu} a_{1-f d}\right) e^{-2 h \xi}}{\left(\xi+\frac{\mu}{\mu_{g}} a_{1-f d}\right)\left(\frac{\mu_{g}}{\mu} a_{1-f d}+\frac{\xi}{\tau_{f d^{2}}}\right)} d \xi
\end{aligned}
$$

Neste artigo os resultados de cálculo da impedância longitudinal e admitância transversal por unidade de comprimento foram obtidos por integração numérica das expressões na sua forma integral via quadratura adaptativa de GaussLobatto - com erro de truncamento de $10^{-6}$ e taxa de amostragem de 10 pontos por década de frequência.

\subsection{Admitância transversal por unidade de comprimento - Métodos de cálculo aproximado}

A admitância transversal calculada na condição de condutor ideal, solo como plano condutor perfeito e tendo em conta as suposições de geometria e de propagação citadas tem como resultado a expressão (18) . A capacitância transversal por unidade de comprimento nestas condições independe da frequência (19) :

$$
\begin{gathered}
Y_{e x t}=i \omega 2 \pi \varepsilon\left[\ln \left(\frac{2 h}{R_{1}}\right)\right]^{-1} \\
C_{e x t}=2 \pi \varepsilon\left[\ln \left(\frac{2 h}{R_{1}}\right)\right]^{-1}
\end{gathered}
$$

onde: $\epsilon$ é a permissividade elétrica do ar (assumida aproximadamente igual à do vácuo);

Porém, na faixa de frequência de 0 a $2 \mathrm{MHz}$ em função da dependência de $\sigma_{g}+\mathrm{i} \omega \epsilon_{g}$ com a frequência torna-se evidente a necessidade de uma reavaliação da admitância transversal de retorno pelo solo.

A correção devido ao retorno pelo solo na admitância transversal pode ser feita de forma aproximada pelo método de aproximações sucessivas de Arismunandar modificado (ARISM-M) ou a partir da integração dos termos de correção de Carson/Wise/Nakagawa modificados (C/W/N-M). Convém ainda destacar o método aproximado de Tesche que relaciona o cálculo da admitância de retorno com o cálculo da impedância de retorno pelo solo, $Z_{g}$ (Tesche, 1992): 


$$
Y_{g}(T-M) \cong \frac{\gamma_{g}^{2}}{Z_{g}}
$$

Desta forma, a admitância transversal resultante pode ser obtida por associação em série entre a admitância transversal calculada na condição de solo ideal (18) e a admitância transversal de retorno pelo solo (20) , $Y_{g}$, (Rachidi et ali, 1996):

$$
Y_{c}=\frac{Y_{e x t} Y_{g}}{Y_{e x t}+Y_{g}}
$$

Neste artigo foram avaliadas as seguintes possibilidades de cálculo aproximado da admitância transversal por unidade de comprimento:

Solo ideal - Admitância transversal por unidade de comprimento obtida a partir do método das imagens e na condição de solo e condutores ideais, $Y_{e x t},(18)$;

S/T-M - Neste modelo a admitância transversal por unidade de comprimento corrigida devido a dependência dos parâmetros do solo com a frequiência - $Y_{c}(S / T-M)$ - foi obtida por associação série entre a admitância de retorno pelo solo $Y_{g}(S / T-M)$ (20) e $Y_{\text {ext }}(18)$. Na expressão (20) a impedância de retorno pelo solo foi obtida através da aproximação de Sunde modificada (8) .

Deri/T-M - Neste modelo a admitância transversal corrigida devido a dependência dos parâmetros do solo com a freqüência $-Y_{c}($ Deri/T-M $)$ - foi obtida por associação série entre a admitância de retorno pelo solo $\mathrm{Y}_{g}($ Deri/T-M) (20) e $\mathrm{Y}_{\text {ext }}(18)$.Na expressão (20) a impedância de retorno pelo solo foi obtida através do método do plano complexo modificado (7) .

C/T-M - Neste modelo a admitância transversal corrigida devido a dependência dos parâmetros do solo com a freqüência - $Y_{c}(C / T-M)$ - foi obtida por associação série entre a admitância de retorno pelo solo $Y_{g}(C / T-M)(20)$ e $Y_{e x t}(18)$ Na expressão (20) a impedância de retorno pelo solo foi obtida por integração numérica da expressão de Carson em seu formato original (14 com propagação axial nula, ou seja, $\left.\gamma_{0}=0\right)$.

C/W/N/T-M - Neste modelo a admitância transversal corrigida devido a dependência dos parâmetros do solo com a freqüência $-Y_{c}(C / W / N / T-M)$ - foi obtida por associação série entre a admitância de retorno pelo solo $Y_{g}(C / W / N / T-M)$ (20) e $\mathrm{Y}_{\text {ext }}(18)$. Na expressão (20) a impedância de retorno pelo solo foi obtida por integração numérica da expressão de Carson modificada (14) .

ARISM-M: Neste modelo a admitância transversal corrigida devido ao retorno pelo solo com parâmetros dependentes da frequiência - $Y_{c}(A R I S M-M)$ - foi obtida a partir do método de aproximações de Arismunandar modificada.

Os resultados obtidos na avaliação da admitância transversal corrigida através dos métodos aproximados descritos foram comparados com a admitância transversal corrigida a partir da integração numérica da expressão de Carson/Wise/Nakagawa modificada, $\boldsymbol{C} / \boldsymbol{W} / \boldsymbol{N}-\boldsymbol{M}$ (17.a). Neste cálculo incide, além das aproximações devido às hipóteses descritas na Seção 2.1, a aproximação de modelagem em relação a altura do cabo condutor e do raio $\left(h>>R_{1}\right)$ e o erro de truncamento do algoritmo de integração.

\section{RESULTADOS}

Os resultados aqui apresentados foram obtidos considerando um cabo condutor grosbeak à uma altura média de 10 metros acima do solo, com as seguintes características: Raio interno (raio da alma de aço), $R_{0}: 4,635 \mathrm{~mm}$; Raio externo (raio do condutor), $R_{1}$ : $12,57 \mathrm{~mm}$; Resistência do cabo condutor em corrente contínua por unidade de comprimento a $75^{\circ} \mathrm{C}, R_{c c}: 0,089898 \Omega / \mathrm{km}$; Permissividade relativa, $\epsilon_{r}: 1$; Permeabilidade magnética relativa, $\mu_{r}: 1$. Foram comparados os diferentes modelos para o cálculo da impedância longitudinal e admitância transversal de retorno descritos anteriormente para casos de solos de baixa e alta resistividade mostrados na Tabela 1 (Portela, 1999). Estes valores foram escolhidos para representar solos de baixa resistividade e alta resistividade, permitindo que se faça uma análise de sensibilidade da influência dos parâmetros do solo no cálculo dos parâmetros da linha.

Tabela 1: Solos de baixa e alta resistividade

\begin{tabular}{|l|l|l|l|l|}
\hline \multirow{2}{*}{ Parâmetros } & \multicolumn{2}{|l|}{ Solo de baixa resistividade } & \multicolumn{2}{|c|}{ Solo de alta resistividade } \\
\cline { 2 - 5 } & constante & " $f d "$ & constante & " $f d "$ \\
\hline$K_{0}[\mu \mathrm{S} / \mathrm{m}]$ & 1700 & 1700 & 50 & 50 \\
\hline$K_{1}\left[\mu \mathrm{S} / \mathrm{m}_{\mathrm{s}}-1\right]$ & 0 & 0,9 & 0 & 0,0021 \\
\hline$\alpha_{1}$ & 0 & 0,62 & 0 & 0,82 \\
\hline
\end{tabular}

\subsection{Impedâncias longitudinais por unidade de comprimento}

Nas Figuras 1 e 2 estão representadas em escala logarítmica a resistência interna por unidade de comprimento, a resistência de retorno pelo solo por unidade de comprimento e a resistência total por unidade de comprimento para casos de solo de baixa resistividade e de alta resistividade, respectivamente. São comparadas as resistências de retorno pelo solo obtidas através das formulações aproximadas modificadas (S-M, DERI-M) e através de integração numérica das formulações de Carson (C-M) e Carson/Wise/Nakagawa modificadas (C/W/N-M). Nas 
Figuras 3 e 4 estão representados os gráficos em escala linear somente da resistência de retorno pelo solo, comparando os diferentes métodos de avaliação (S-M, Deri-M, C-M, $\mathrm{C} / \mathrm{W} / \mathrm{N}-\mathrm{M}$ ), para casos de baixa resistividade e alta resistividade do solo, respectivamente.

Observa-se que a resistência interna é constante até $100 \mathrm{~Hz}$ e varia com a frequência devido ao efeito pelicular - que a partir de $100 \mathrm{~Hz}$ diminui a área efetiva de condução do condutor e, consequentemente, resulta no aumento da resistência interna com a frequência. Em baixas frequências (até $100 \mathrm{~Hz}$ ) a contribuição da resistência interna na resistência total é maior do que a contribuição da parcela de retorno pelo solo. Por exemplo, em $60 \mathrm{~Hz}$, o valor da resistência interna calculada é de $0,0912 \Omega / \mathrm{km}$ e o valor da resistência de retorno pelo solo de $0,0599 \Omega / \mathrm{km}$ resultando na resistência total (soma da resistência interna com a de retorno pelo solo) de $0,15 \Omega / \mathrm{km}$.

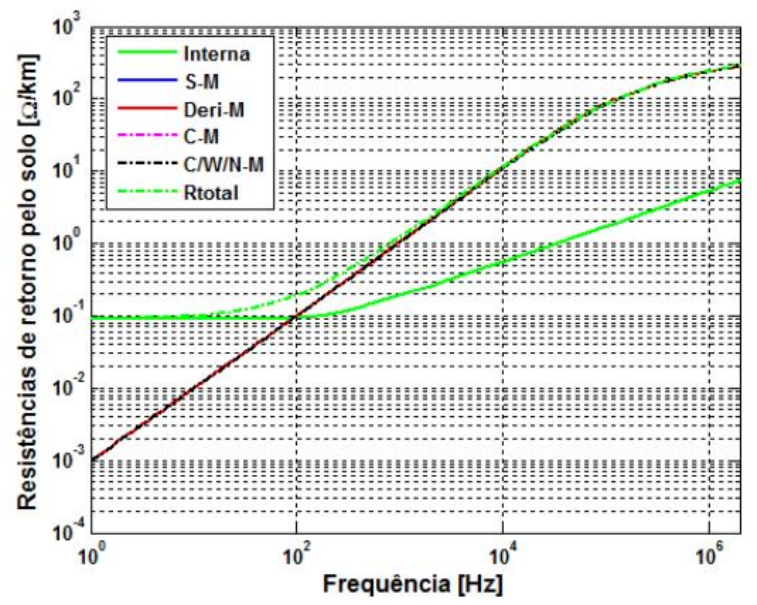

Figura 1: Resistência interna, de retorno pelo solo e total por unidade de comprimento em escala logarítmica: baixa

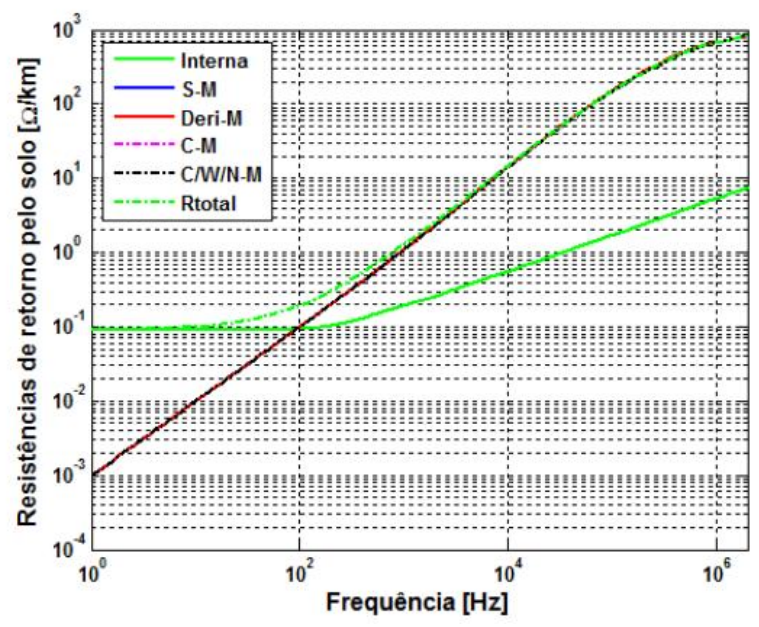

Figura 2: Resistência interna, de retorno pelo solo e total por unidade de comprimento em escala logarítmica: alta resistividade.
Comparando os casos de solo de baixa resistividade (Figuras 1 e 3) e alta resistividade (Figuras 2 e 4), verifica-se que até $1 \mathrm{kHz}$ as resistências de retorno por unidade de comprimento para solos de baixa e de alta resistividade têm a mesma ordem de grandeza. Porém, a medida que a frequência aumenta, verifica-se uma maior influência dos parâmetros do solo com a frequência nas resistências de retorno para solos de alta resistividade. Neste caso de alta resistividade a resistência de retorno varia de $14,12 \Omega / \mathrm{km}$ em $10 \mathrm{kHz}$ a $812 \Omega / \mathrm{km}$ em $2 \mathrm{MHz}$, enquanto que para solos de baixa resistividade varia de $11 \Omega / \mathrm{km}$ em $10 \mathrm{kHz}$ a $284 \Omega / \mathrm{km}$ em $2 \mathrm{MHz}$.

Comparando as diferentes formulações utilizadas verifica-se que as formulações aproximadas e modificadas de Sunde (S-M) e da Deri (DERI-M) apresentam valores idênticos, como esperado, já que as duas expressões são equivalentes. O comportamento assintótico das formulações DERI-M e SM em relação às formulações de Carson (C-M e C/W/N-M) é observado na faixa de $1 \mathrm{kHz}$ a $100 \mathrm{kHz}$ (Figura 3) e na faixa de $1 \mathrm{kHz}$ a $1 \mathrm{MHz}$ (Figura 4). A formulação Deri-M apresenta valores de impedância de retorno ligeiramente superiores ao caso C/W/N-M. Entre $1 \mathrm{MHz}$ e $2 \mathrm{MHz}$ todas as formulações apresentam os mesmos valores devido ao comportamento assintótico das formulações aproximadas. $\mathrm{O}$ erro assintótico deve ser avaliado para o caso de linha trifásica.

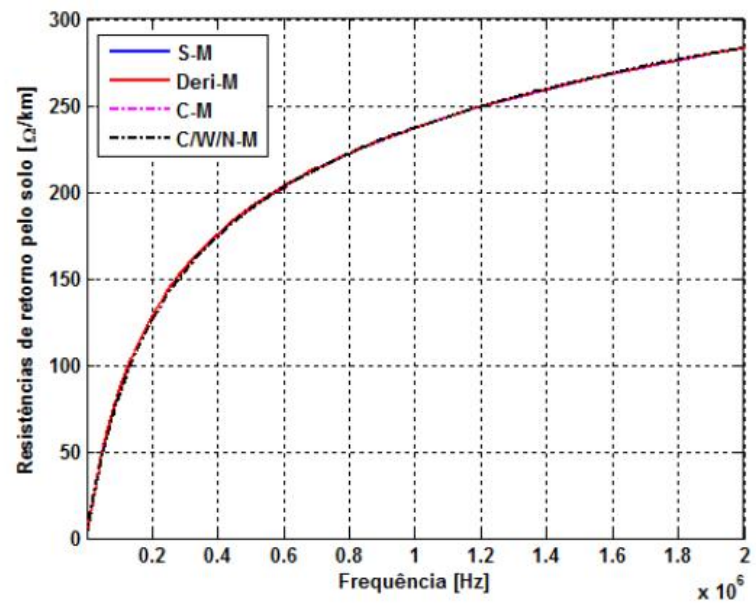

Figura 3: Resistência de retorno pelo solo por unidade de comprimento em escala linear: baixa resistividade.

Na Figura 5 está representada a relação entre a resistência de retorno pelo solo e a resistência interna. Em $100 \mathrm{~Hz}$ a resistência interna e a de retorno pelo solo têm a mesma ordem de grandeza, e a partir desse valor de frequência a resistência de retorno pelo solo apresenta valores cada vez maiores com contribuição predominante na resistência total em relação à interna acima de $10 \mathrm{kHz}$. 


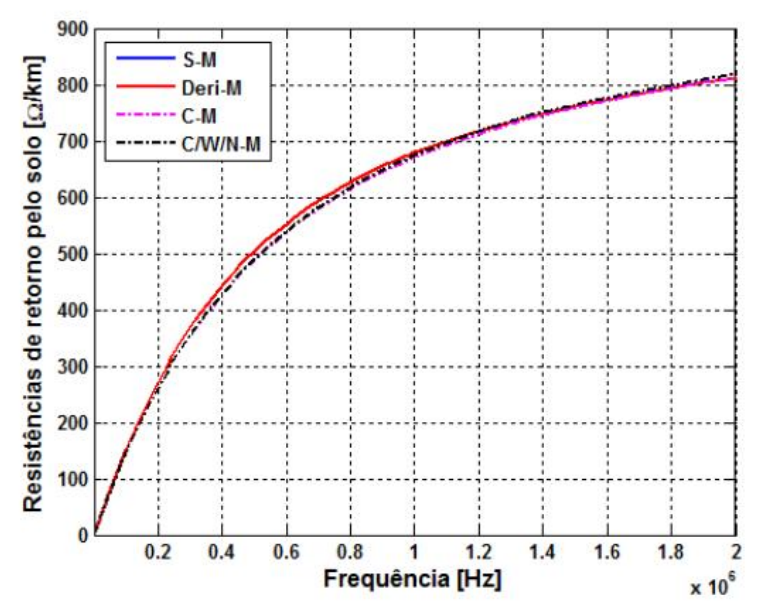

Figura 4: Resistência de retorno pelo solo por unidade de comprimento em escala linear: alta resistividade.

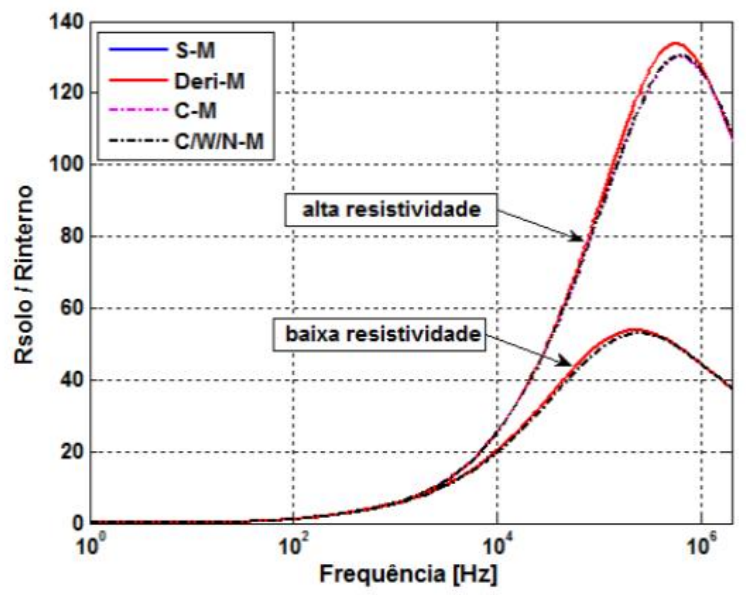

Figura 5: Relação entre resistência do solo e resistência interna: comparação para diferentes formulações de cálculo.

Nota-se que para solos de baixa resistividade na faixa de $1 \mathrm{~Hz}$ a $100 \mathrm{~Hz}$ a relação entre a resistência de retorno pela resistência interna varia de 0,01 a 1,07. Para freqüências superiores tem valor máximo de $53,1 \mathrm{em} 244 \mathrm{kHz}$ e diminui para, 38 vezes em $2 \mathrm{MHz}$. Para solo de alta resistividade essa relação tem a mesma ordem de grandeza que o caso de solos de baixa resistividade até $3 \mathrm{kHz}$, porém, em altas frequências a relação atinge valor máximo de 134 vezes em $568 \mathrm{kHz}$ e volta a diminuir para 110 vezes em $2 \mathrm{MHz}$. Para frequências acima de $10 \mathrm{kHz}$ a contribuição da resistência de retorno pelo solo é muito maior que a interna (representa $96 \%$ da resistência total enquanto a interna situa-se abaixo $\operatorname{dos} 5 \%)$.

Nas Figuras 6 a 9 estão representados análises de sensibilidade do módulo e do argumento da impedância de retorno para três casos distintos de representação dos parâmetros do solo no cálculo da resistência de retorno pelo solo: (i) solo com condutividade $\left(\sigma_{g}\right)$ constante e independente da frequência e $\omega \epsilon_{g}$ nulo; (ii) solo com $\sigma_{g}$ constante mais uma parcela com dependência da frequência hipoteticamente idêntica à parcela que contribuiria para o aumento da condutividade caso fosse considerado o "modelo completo", porém, ainda com $\omega \epsilon_{g}$ nulo; (iii) "modelo completo" que considera a dependência de $\sigma_{g}$ e $\omega \epsilon_{g}$ com a frequiência, mas que tem as restrições citadas na Seção 2.1.

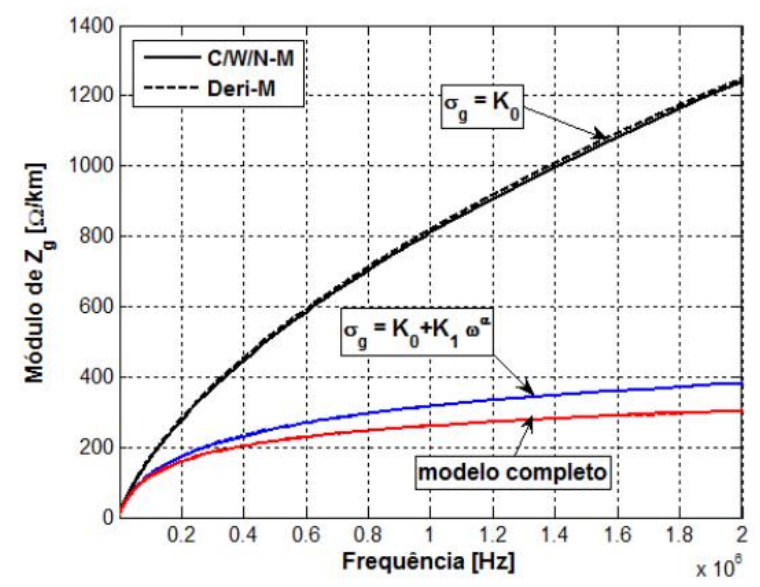

Figura 6: Módulo da impedância de retorno pelo solo por unidade de comprimento: Sensibilidade em relação à dependência de $\sigma_{g}$ e $\omega \epsilon_{g}$ com a frequência: baixa resistividade.

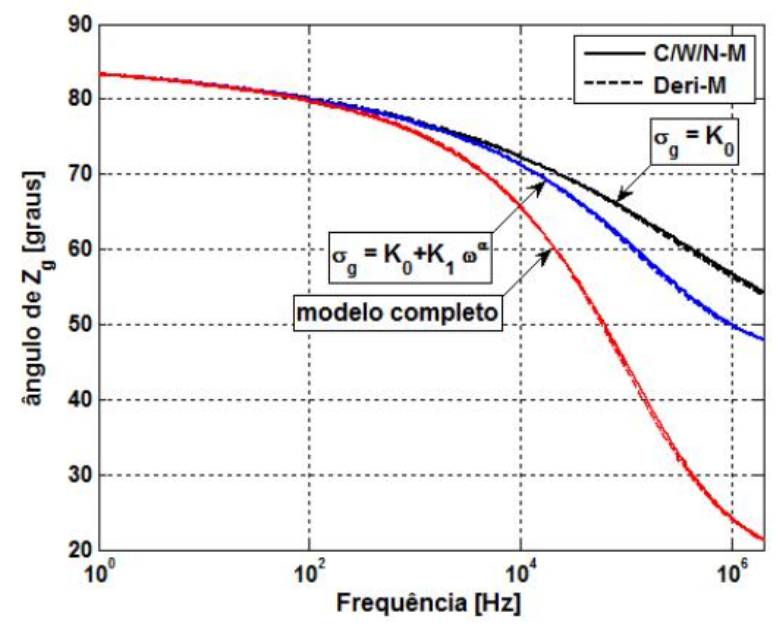

Figura 7: Ângulo da impedância de retorno pelo solo por unidade de comprimento. Sensibilidade em relação à dependência de $\sigma_{g}$ e $\omega \epsilon_{g}$ com a frequência: baixa resistividade.

Notam-se valores de impedância maiores e uma maior contribuição das parcelas que variam com a frequência para o caso de alta resistividade em relação ao caso de baixa resistividade. Os módulos das impedâncias de retorno 
nos casos (i) e (ii) apresentam valores mais elevados em comparação com o caso (iii) - resultado esperado já que o módulo da condutividade é menor nos dois primeiros casos.

Observa-se que o argumento da impedância de retorno pelo solo tende a valores baixos (muito menores que os casos i e ii - Figuras 7 e 9) ao se incluir o efeito da permissividade do solo (caso iii), o que implica numa redução relativa do efeito do solo na resistência de retorno (Figuras 10 e 11).

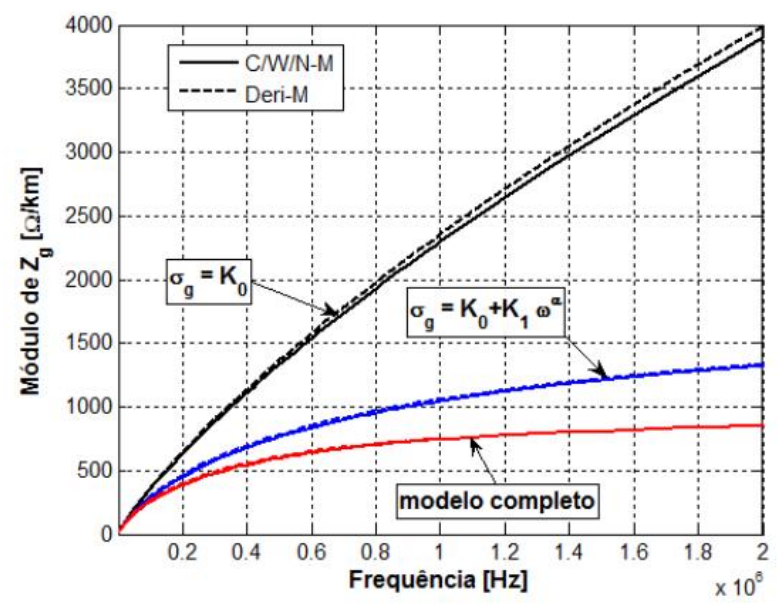

Figura 8: Módulo da Impedância de retorno pelo solo por unidade de comprimento. Sensibilidade em relação à dependência de $\sigma_{g}$ e $\omega \epsilon_{g}$ em função da frequência: alta resistividade.

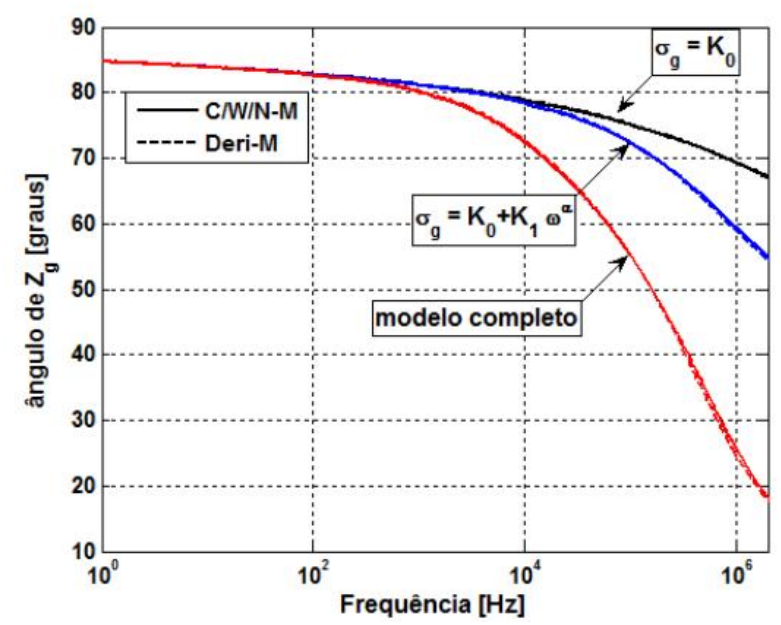

Figura 9: Ângulo da impedância de retorno pelo solo por unidade de comprimento. Sensibilidade em relação à dependência de $\sigma_{g}$ e $\omega \epsilon_{g}$ em função da frequência: alta resistividade.

Nas Figuras 10 e 11 estão representados os gráficos da resistência de retorno pelo solo para três casos distintos de representação dos parâmetros do solo para o cálculo da resistência de retorno pelo solo apresentados anteriores: (i), (ii) e (iii), respectivamente.

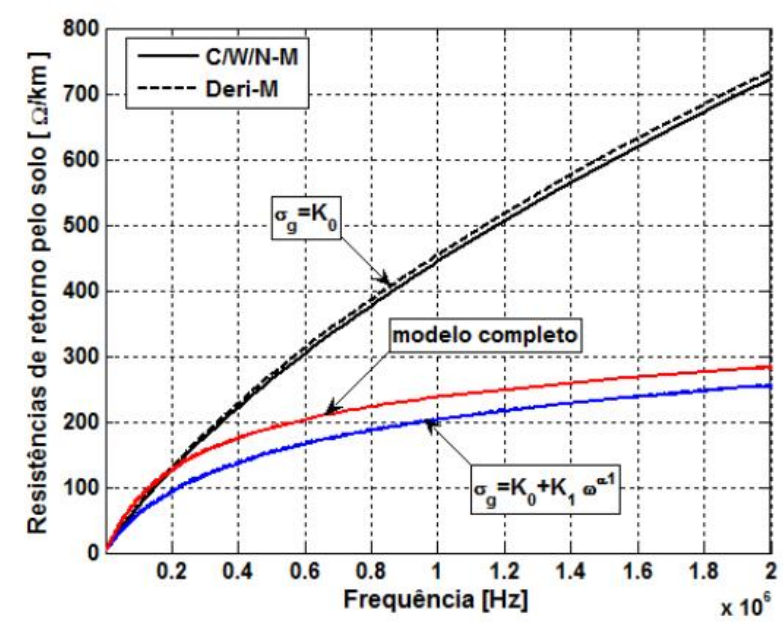

Figura 10: Resistência de retorno pelo solo por unidade de comprimento. Sensibilidade em relação à dependência de $\sigma_{g}$ e $\omega \epsilon_{g}$ em função da frequência: baixa resistividade.

É evidente que a diferença entre o caso (ii) e o (iii) é a contribuição de $\omega \epsilon_{g}$ na resistência de retorno pelo solo, enquanto que a diferença entre o caso (ii) e o (i) $\left(\sigma_{g}\right.$ constante) é a contribuição da parcela de $\sigma_{g}$ que depende da frequência. Observa-se que o caso (iii) apresenta valores de resistências maiores em relação aos casos (i) e (ii) - que são muito próximos até $100 \mathrm{kHz}$. Na faixa de $100 \mathrm{kHz}$ a $2 \mathrm{MHz}$ a inclusão da parcela de $\sigma_{g}$ que depende da frequência, caso (ii), resulta numa diminuição da resistência de retorno pelo solo em relação ao caso (i). Porém, ao se incluir o termo $\omega \epsilon_{g}$, caso (iii), a resistência de retorno apresenta valores maiores em relação ao caso (ii), um resultado não intuitivo, mas que é explicado pela redução significativa do argumento da impedância de retorno pelo solo.

Nas Figuras 12 a 16 foram analisadas as implicações da dependência dos parâmetros do solo com a frequência no cálculo da indutância total por unidade de comprimento. Foram feitas comparações entre os métodos aproximados (S-M e DERI-M) e o cálculo efetuado por integração das formulações de Carson (C-M e C/W/N-M).

Nota-se que a indutância interna tem comportamento inverso em relação à resistência, é constante $(0,05 \mathrm{mH} / \mathrm{km})$ para baixas frequências e diminui consideravelmente com a frequência a partir de $100 \mathrm{~Hz}$. Isso justifica-se pelo fato da indutância interna ser proporcional à área efetiva de condução que diminui à medida que a frequência aumenta devido ao efeito pelicular (Figura 12). A indutância externa na condição de solo ideal é constante $(1,47 \mathrm{mH} / \mathrm{km})$ em toda a faixa de frequência. Tanto a indutância interna quanto a de retorno pelo solo diminuem com o aumento da frequência, ou 


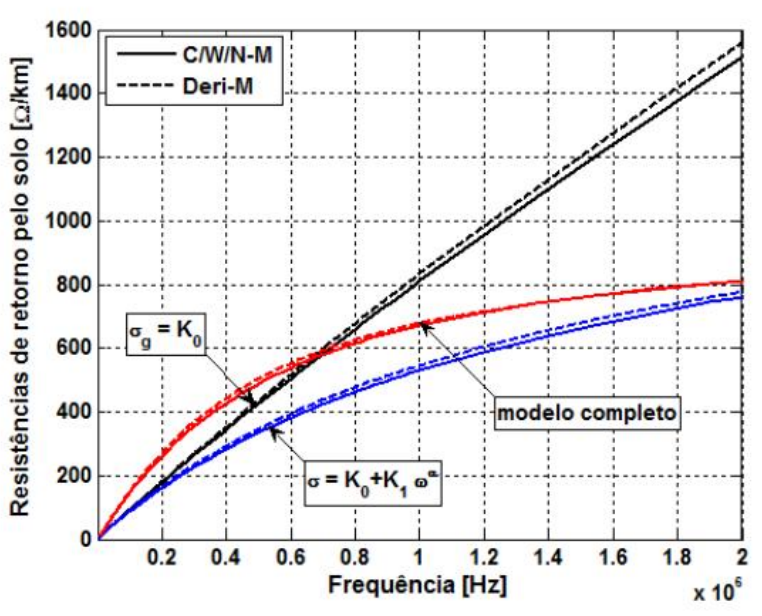

Figura 11: Resistência de retorno pelo solo por unidade de comprimento. Sensibilidade em relação à variação de $\sigma_{g} \mathrm{e}$ $\omega \epsilon_{g}$ em função da frequência: alta resistividade.

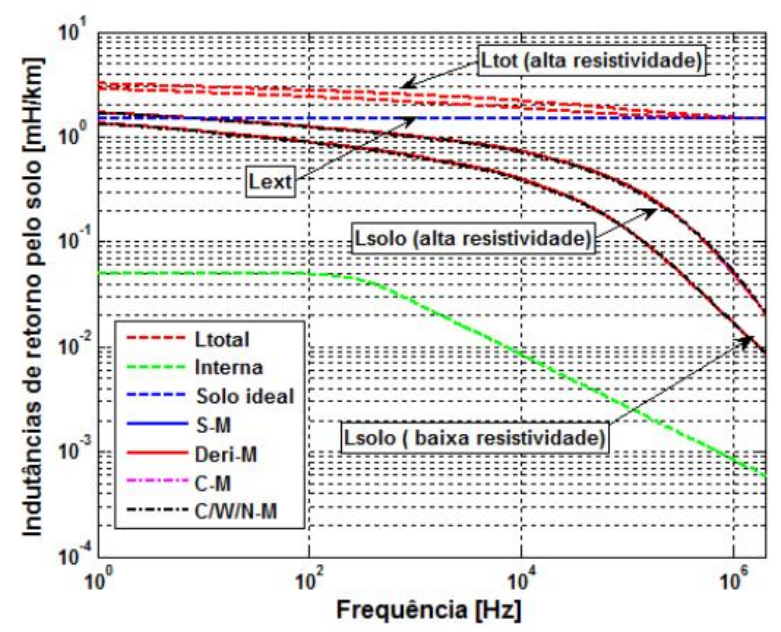

Figura 12: Indutância interna, de retorno pelo solo e total por unidade de comprimento.

seja, a indutância total tende ao valor da indutância externa para frequências elevadas.

Na Figura 13 está representada a relação entre a indutância de retorno pelo solo e a indutância interna. Observa-se que a contribuição da indutância de retorno pelo solo é muito maior que a da indutância interna em toda a faixa de frequência, tanto para os exemplos de solos de baixa quanto para alta resistividade.

A relação entre a indutância de retorno pelo solo e a indutância externa (Figura 14) varia de 0,9 a 0,6 vezes entre 0 a $100 \mathrm{~Hz}$ para solo de baixa resistividade, os termos são da mesma ordem de grandeza. Na faixa de $100 \mathrm{~Hz}$ a $10 \mathrm{kHz}$ a relação entre a indutância do solo e a indutância interna é da

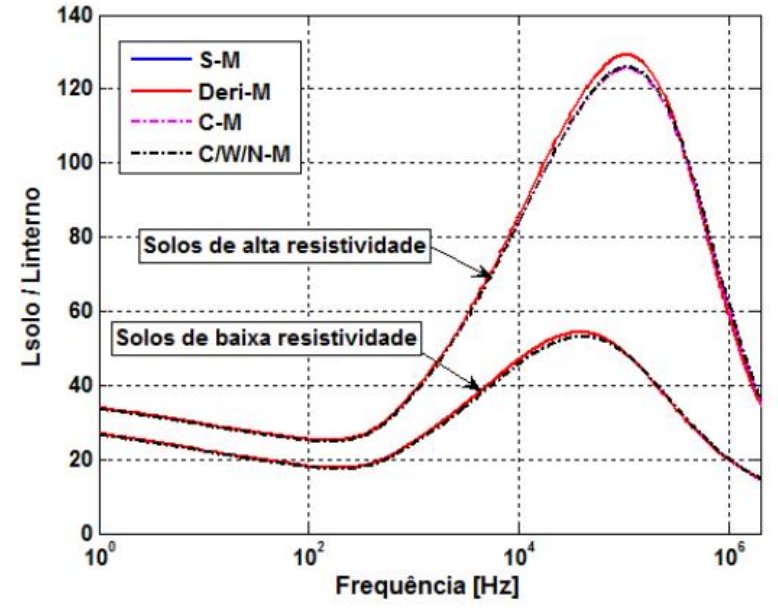

Figura 13: Relação entre indutância de retorno pelo solo e indutância interna considerando diferentes formulações de cálculo.

ordem de 0,6 a 0,3 . De $10 \mathrm{kHz}$ a $2 \mathrm{MHz}$ esta relação varia de 0,3 a valores da ordem de $10^{-2}$

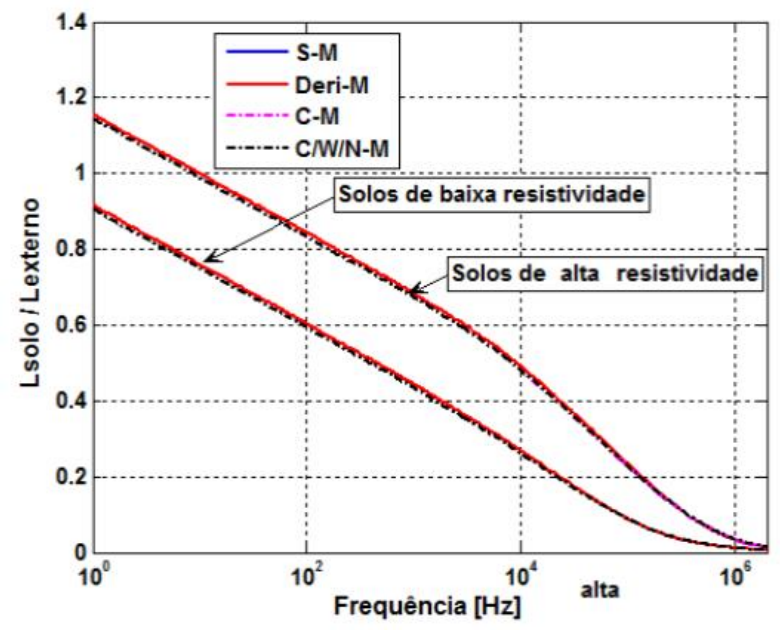

Figura 14: Relação entre indutância de retorno pelo solo e indutância externa (solo ideal).

Portanto, para altas frequências (acima de $10 \mathrm{kHz}$ ) ambas as contribuições internas e solo tornam-se menos significativas o que explica a aproximação das curvas de indutância total e indutância externa. Comparando os casos de baixa e alta resistividade verifica-se uma maior contribuição da indutância de retorno pelo solo na indutância total para o caso de solo de alta resistividade. A indutância interna representa no máximo $2 \%$ da indutância total até $100 \mathrm{~Hz}$ e abaixo de $0,5 \%$ de $10 \mathrm{kHz}$ a $2 \mathrm{MHz}$. A indutância externa varia de $50 \%$ da indutância total em baixa frequência a valores acima de $97 \%$ para frequências acima de $1 \mathrm{MHz}$. 
Os erros assintóticos entre modelos aproximados e o cálculo via integração numérica das formulações de Carson são menores no cálculo da indutância de retorno em relação ao erro assintótico no cálculo da resistência de retorno pelo solo, tanto para os exemplos de solos de baixa resistividade quanto para alta resistividade.

Nas Figuras 15 e 16 estão apresentados os gráficos de sensibilidade para os três casos distintos de representação dos parâmetros do solo para o cálculo da indutância de retorno, para os casos de solo de baixa (Figura 15) e de alta resistividade (Figura 16). Nota-se que em ambos os casos a influência das parcelas dos parâmetros do solo que variam com a frequência são notáveis acima de $1 \mathrm{kHz}$.

Nota-se que para solos de alta resistividade (Figura 16) a influência da dependência de $\sigma_{g}$ quanto de $\omega \epsilon_{g}$ são mais significativas do que no caso de baixa resistividade. As indutâncias de retorno apresentam valores maiores para solo com alta resistividade.

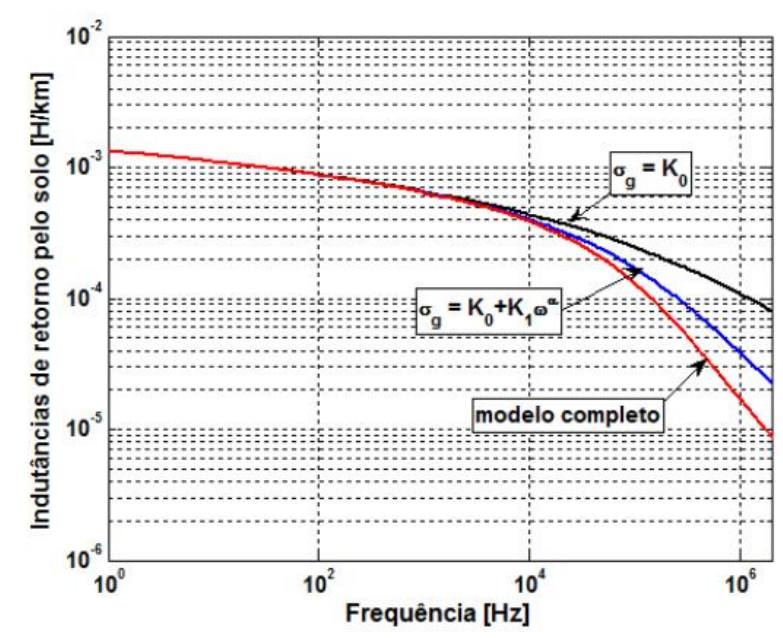

Figura 15: Indutância de retorno pelo solo por unidade de comprimento. Sensibilidade em relação à variação de $\sigma_{g} \mathrm{e}$ $\omega \epsilon_{g}$ em função da frequência: baixa resistividade.

Nas Figuras 17 e 18 estão apresentados os erros percentuais entre o modelo de representação do solo a parâmetros constantes e o "modelo completo" (C/W/N-M) que considera a dependência dos parâmetros do solo com a frequência na avaliação da impedância total por unidade de comprimento para os casos de baixa e alta resistividade, respectivamente. A representação da dependência dos parâmetros do solo com a frequência tem influência significativa na avaliação dos parâmetros longitudinais da linha.

Para a resistência total por unidade de comprimento $\left(R_{t o t}\right)$, observa-se que na faixa de $10 \mathrm{kHz}$, os erros relativos situam-se entre $20 \%$ (baixa resistividade) e $40 \%$ (solos de alta resistividade) e acima de $100 \%$ para este último caso

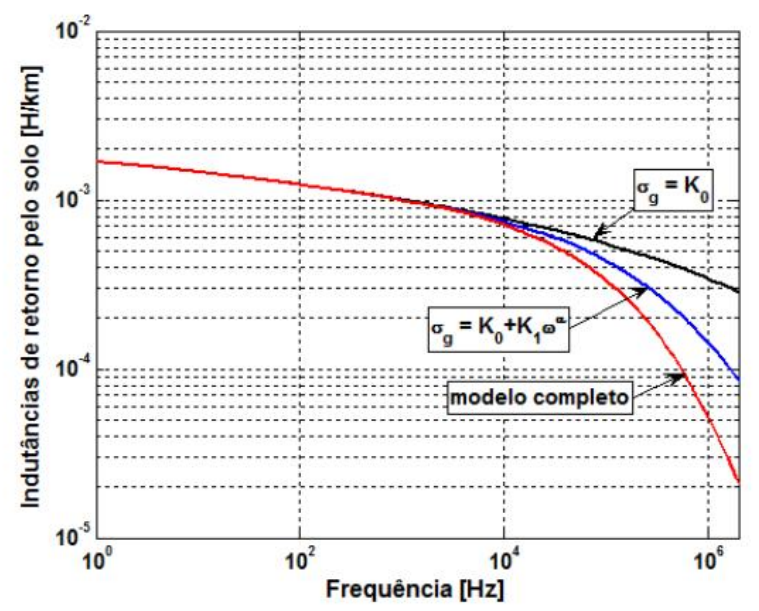

Figura 16: Indutância de retorno pelo solo por unidade de comprimento. Sensibilidade em relação à variação de $\sigma_{g} \mathrm{e}$ $\omega \epsilon_{g}$ em função da frequência: alta resistividade.

na faixa de $1 \mathrm{MHz}$ a $2 \mathrm{MHz}$. Na avaliação da indutância, os erros relativos entre $10 \mathrm{kHz}$ e $2 \mathrm{MHz}$ são menores e situam-se na faixa de 4 a $8 \%$ (baixa resistividade) e de 4 a $19 \%$ (alta resistividade).

\subsection{Capacitâncias transversais unidade de comprimento}

Nas Figura 19 e 20 estão representadas respectivamente a capacitância externa por unidade de comprimento (calculada na condição de solo ideal) e a capacitância de retorno pelo solo por unidade de comprimento para alturas do cabo condutor de 5 e $10 \mathrm{~m}$. Nota-se que a capacitância externa depende da altura do cabo em relação ao solo e do raio externo do mesmo e independe da frequencia. Para este caso uma diminuição de $50 \%$ na altura do condutor representa um aumento de 10,4\% na capacitância externa - constante em toda a faixa de frequência em estudo.

Por outro lado, a capacitância de retorno pelo solo depende da altura do cabo e dos parâmetros do solo (condutividade e permissividade). Neste caso particular, para um aumento de $50 \%$ na altura verifica-se um aumento na capacitância de retorno pelo solo que varia de $7 \%$ (para frequências menores de $1 \mathrm{kHz})$ a $18 \%(2 \mathrm{MHz})$.

A capacitância transversal total por unidade de comprimento é o resultado da associação série entre as capacitância externa na condição de solo ideal (Figura 19) e a de retorno pelo solo (Figura 20).

Observa-se que a capacitância de retorno pelo solo tende a valores elevados - em comparação com a capacitância externa (condição de solo ideal) - para frequências menores 


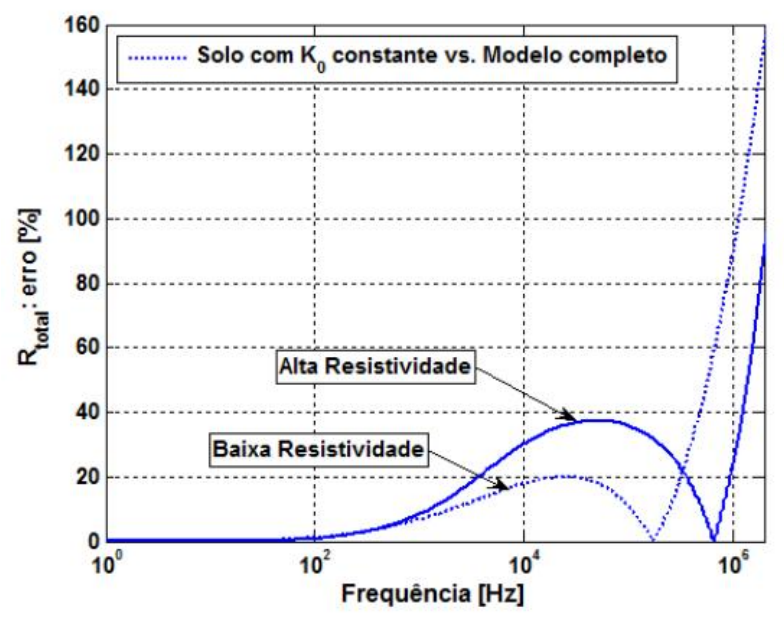

Figura 17: Resistência total por unidade de comprimento: Erro relativo entre modelo de solo a parâmetros constantes e modelo de solo com parâmetros dependentes da frequência.

que $100 \mathrm{~Hz}$ e acima de $200 \mathrm{kHz}$ (caso de alta resistividade). Ou seja, a capacitância transversal total por unidade de comprimento tende a ser igual à capacitância externa (condição de solo ideal) nas faixas de frequências mencionas.

Nas Figuras 21 e 22 estão representados os gráficos da capacitância total por unidade de comprimento em escala logarítmica comparando os diferentes modelos de cálculo descritos para os casos de solo de baixa e alta resistividade, respectivamente.

Pôde-se verificar que os modelos aproximados apresentam resultados próximos em toda a faixa de frequência. Nas expressões aproximadas C/T-M e C/W/N/T-M a impedância de retorno pelo solo foi obtida por integração numérica. Os métodos de avaliação $\mathbf{S} / \mathbf{T}-\mathbf{M}$ e DERI/T-M são equivalentes e consequentemente apresentam resultados idênticos quando é incluída a dependência dos parâmetros do solo com a frequência.

Nota-se que todos os modelos de cálculo com aproximação de Tesche (20) apresentam resultados do cálculo da capacitância transversal total por unidade de comprimento próximos da condição de solo ideal para caso de baixa resistividade (Figura 21) - onde a contribuição do solo na admitância total é de fato insignificativa. Os valores obtidos apresentam uma pequena variação entre $7,546 \mathrm{nF} / \mathrm{km}$ em baixas frequências e 7,545 nF/km em $10 \mathrm{kHz}$.

O método de cálculo ARISM-M apresenta variação entre $7,546 \mathrm{nF} / \mathrm{km}$ em baixas frequências e 7,540 nF/ km enquanto que o modelo $\mathbf{C} / \mathbf{W} / \mathbf{N}-\mathbf{M}$ apresenta resultado com maior sensibilidade em relação a variação dos parâmetros do solo com a frequência, entre 7,546 nF/km e 7,529 nF/km. Este

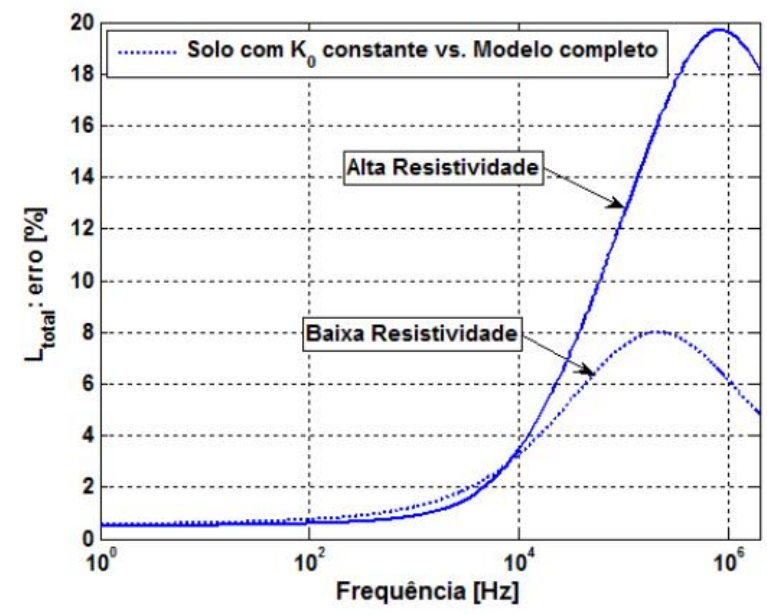

Figura 18: Indutância total por unidade de comprimento: Erro relativo entre modelo de solo a parâmetros constantes e modelo de solo com parâmetros dependentes da frequência.

resultado será utilizado como referência na presente análise. As discrepâncias entre os valores obtidos por C/W/N-M e ARISM-M são insignificantes, da ordem de $10^{-2}$ a $10^{-3}$.

Para solo de alta resistividade (Figura 22) verifica-se uma diferença maior entre os modelos com a aproximação de Tesche em relação aos modelos ARISM-M e C/W/N/T-M e uma maior sensibilidade em relação à dependência dos parâmetros do solo com a frequência.

Tendo o modelo $\mathbf{C} / \mathbf{W} / \mathbf{N}-\mathbf{M}$ como referência, verifica-se que para o caso de alta resistividade os modelos com a aproximação de Tesche não representam de forma adequada a contribuição do solo. A capacitância calculada com os modelos com os referidos modelos apresenta variação máxima entre 7,546 nF/km em baixas frequências e $7,518 \mathrm{nF} / \mathrm{km}$ a $40 \mathrm{kHz}$. A capacitância calculada através do modelo ARISM-M possui uma variação máxima entre $7,546 \mathrm{nF} / \mathrm{km}$ em baixas frequências e 7,455 nF/km (100 kHz) enquanto que o modelo $\mathbf{C} / \mathbf{W} / \mathbf{N}-\mathbf{M}$ apresenta uma variação maior de 7,546 nF/km para 7,418 nF/km $(100 \mathrm{kHz})$.

Porém, nota-se que os diferentes modelos de cálculo apresentam resultados com o mesmo comportamento em altas frequências (acima de $200 \mathrm{kHz}$ ), o que pode ser observado nas Figuras 23 e 24, para os casos de solo de baixa resistividade e de alta resistividade, respectivamente.

Entre os modelos de cálculo aproximado o que mais se aproxima do cálculo com as formulações de Carson (C/W/N-M) é o de Arismunandar modificado (ARISM-M). Mesmo assim, para casos de alta resistividade há uma pequena diferença de $0,5 \%$ entre os dois procedimentos de cálculo que deve ser avaliada para o caso de linha trifásica. 


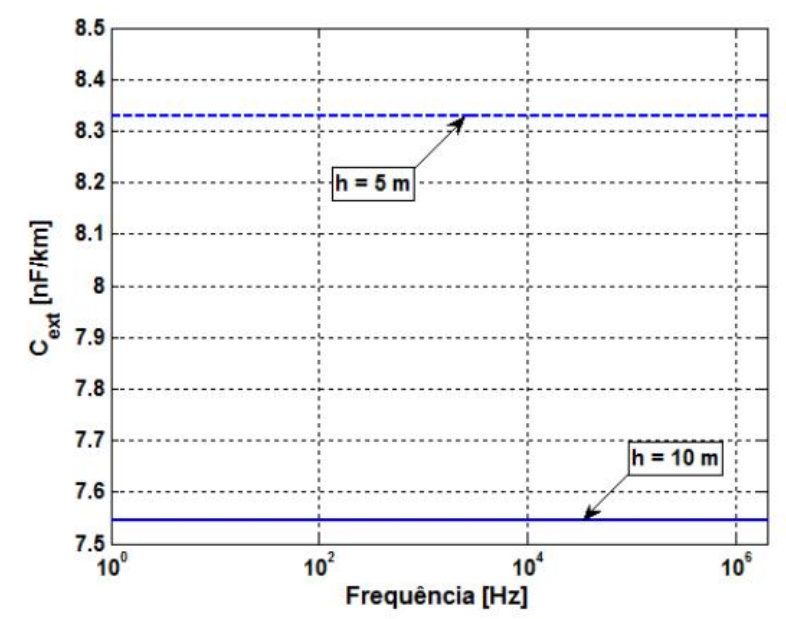

Figura 19: Sensibilidade da capacitância externa por unidade de comprimento (solo ideal) para uma variação de 50 \% na altura do condutor.

Nas Figuras 25 e 26 estão representados os gráficos de análise de sensibilidade para três casos distintos de representação dos parâmetros do solo no cálculo da capacitância transversal total por unidade de comprimento, em comparação com o caso de representação de solo ideal. Esta análise é feita para solo de baixa resistividade (Figura 25) e alta resistividade (Figura 26).

Na Figura 27 pode-se observar que o erro percentual entre a representação tradicional com solo ideal e o modelo do solo com dependência da freqüência, C/W/N-M, varia de $0,23 \%$ em $2 \mathrm{MHz}$ para solos de baixa resistividade a $1,7 \%$ em $118 \mathrm{kHz}$ para solos de alta resistividade.

Dos resultados apresentados nas Figuras 28 e 29 conclui-se que a parcela de $\sigma_{g}$ que depende da frequência tem influência significativa no cálculo da capacitância transversal total por unidade de comprimento. O "modelo completo - C/W/N-M" aproxima-se da condição de "solo ideal" com o aumento da condutividade devido à dependência deste parâmetro com a frequência, e quando não representada induz a um erro de modelagem considerável - máximo de $1,7 \%$ em 1,36 MHz para solos de baixa resistividade e $14,1 \%$ em $370 \mathrm{kHz}$ para solos de alta resistividade, Figura 28.

Nota-se também que a dependência de $\omega \epsilon_{g}$ (que está relacionada com a densidade de corrente de deslocamento induzida no solo) não tem muita influência no resultado quando comparado com o "modelo completo-C/W/N-M". A ausência da dependência de $\omega \epsilon_{g}$ nos gráficos da Figura 29 resulta em erro percentual de $0,1 \%$ em $2 \mathrm{MHz}$ para solo de baixa resistividade e de 2,82\% (em 1,36 MHz) para solo de alta resistividade.

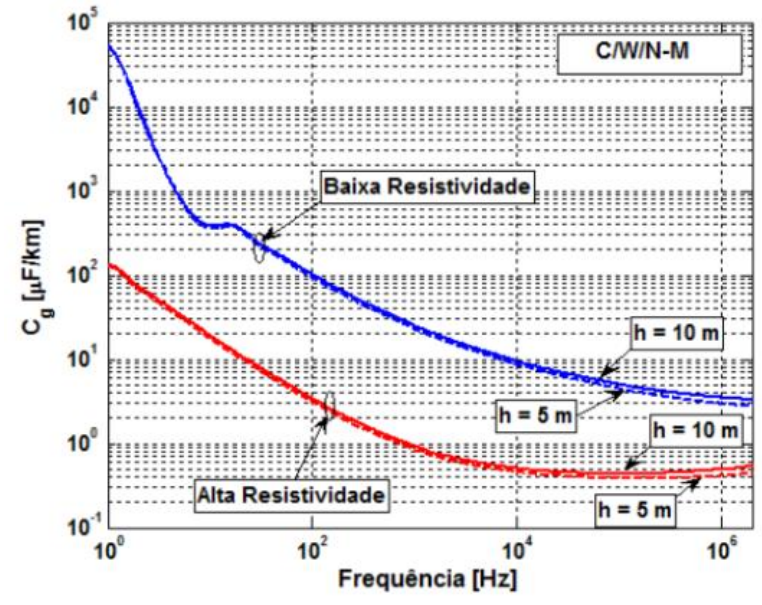

Figura 20: Sensibilidade da capacitância de retorno pelo solo para uma variação de $50 \%$ na altura do condutor: comparação para solo de baixa e alta resistividade.

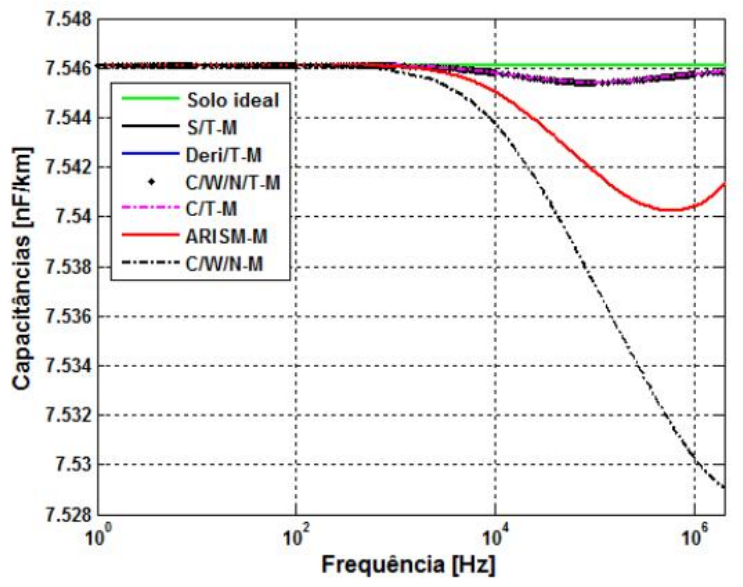

Figura 21: Capacitância por unidade de comprimento: baixa resistividade.

\section{CONCLUSÕES}

Neste artigo foi apresentada uma análise dos parâmetros elétricos de uma linha formada por um único condutor acima de um solo condutor imperfeito, onde a impedância longitudinal e a admitância transversal de retorno pelo solo foram avaliadas através de métodos tradicionais de cálculo e através da integração numérica das formulações de Carson modificadas considerando a dependência da condutividade e de $\omega \epsilon_{g}$ com a frequência.

A dependência dos parâmetros do solo com a frequência têm influência significativa no módulo e argumento da impedância de retorno pelo solo e, consequentemente, na impedância longitudinal total por unidade de comprimento da linha. 


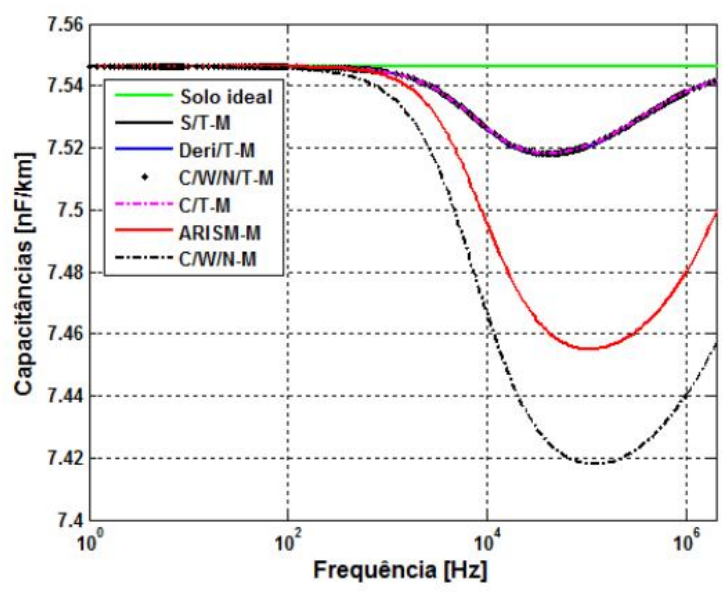

Figura 22: Capacitância por unidade de comprimento: alta resistividade.

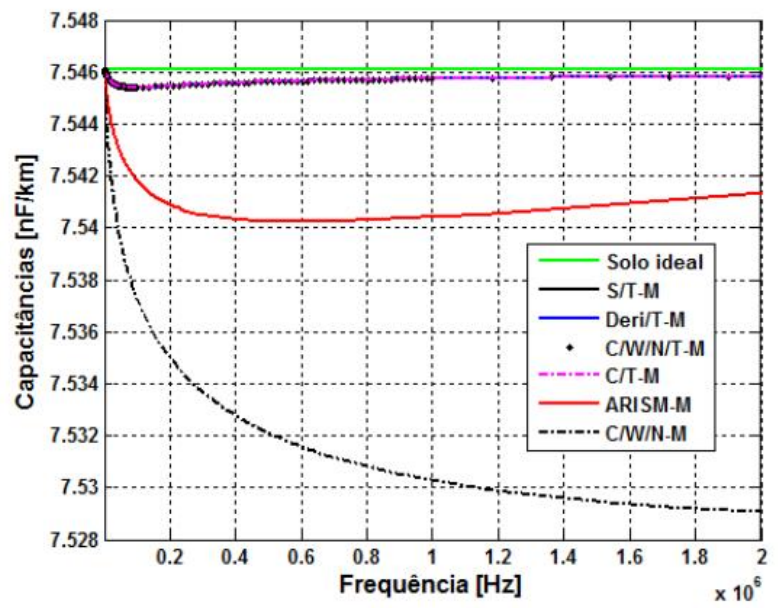

Figura 23: Capacitância por unidade de comprimento em escala linear: baixa resistividade.

$\mathrm{Na}$ resistência total por unidade de comprimento, os erros relativos máximos variam entre $20 \%$ (solos de baixa resistividade) e $40 \%$ (solos de alta resistividade) e são elevados (acima de $40 \%$ ) para este último caso na faixa de $1 \mathrm{MHz}$ a $2 \mathrm{MHz}$. Na avaliação da indutância, os erros relativos entre $10 \mathrm{kHz}$ e $2 \mathrm{MHz}$ são menores e variam de 4 a $8 \%$ (baixa resistividade) e de 4 a $19 \%$ (alta resistividade). As faixas de frequências mencionadas cobrem os transitórios de manobra e transitórios rápidos ocasionados por descargas atmosféricas. As diferenças observadas alteram os parâmetros de propagação da linha nas faixas de frequência mencionadas e servem de motivação para análise do comportamento da linha durante a ocorrência de transitórios eletromagnéticos considerando a correta modelagem dos parâmetros do solo.

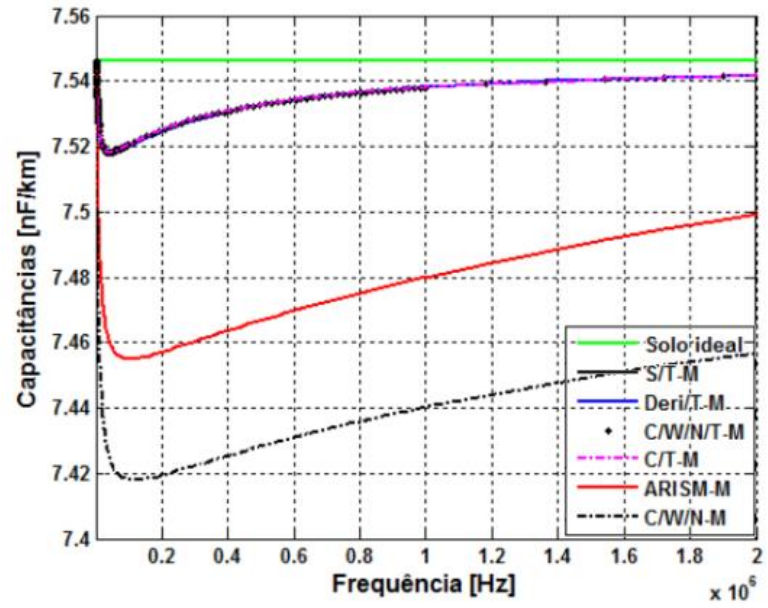

Figura 24: Capacitância por unidade de comprimento em escala linear: alta resistividade.

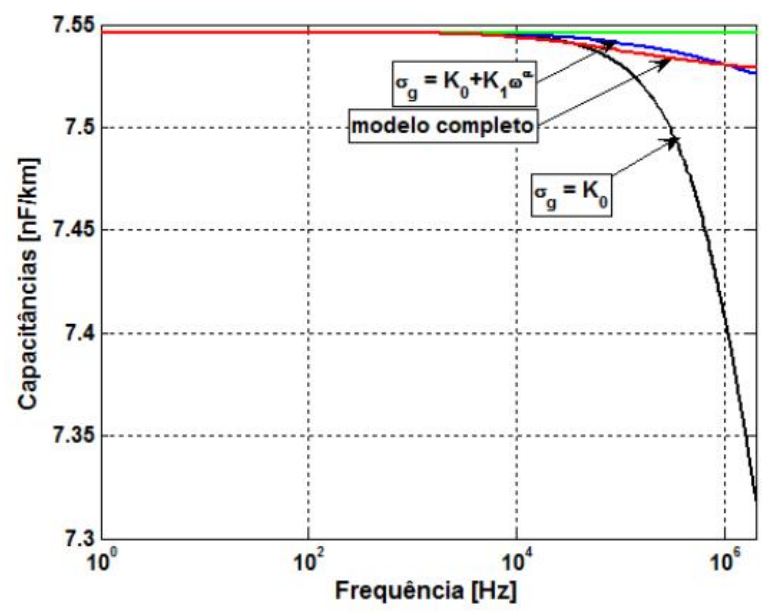

Figura 25: Capacitância por unidade de comprimento: Sensibilidade em relação à variação de $\sigma_{g}$ e $\omega \epsilon_{g}$ em função da frequência: baixa resistividade.

Da análise de sensibilidade da capacitância por unidade de comprimento considerando três casos distintos de representação dos parâmetros do solo (condutividade constante, condutividade variável e condutividade e $\omega \epsilon_{g}$ dependentes da frequência) conclui-se que:

- O erro percentual máximo entre a representação tradicional com solo ideal e o "modelo completo" varia de $0,23 \%$ em $2 \mathrm{MHz}$ para solos de baixa resistividade e $1,7 \%$ em $118 \mathrm{kHz}$ para solos de alta resistividade.

- A representação da dependência de $\omega \epsilon_{g}$ do solo não tem muita influência no cálculo da capacitância por unidade de comprimento quando comparado com o "modelo completo": chega a valores da ordem de $3 \%$ no caso de alta resistividade. 


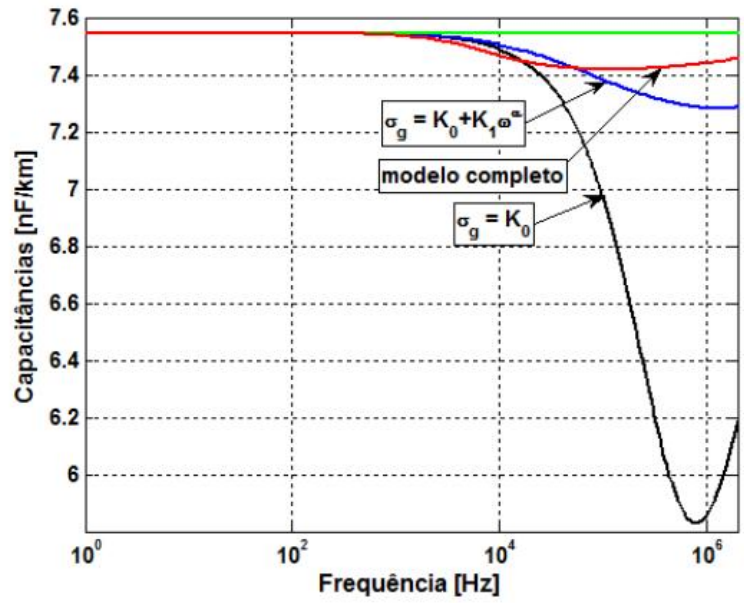

Figura 26: Capacitância por unidade de comprimento: Sensibilidade em relação à variação de $\sigma_{g}$ e $\omega \epsilon_{g}$ em função da frequência: baixa alta resistividade.

- A representação da dependência de $\sigma_{g}$ com a frequência tem grande influência no cálculo da capacitância por unidade de comprimento da linha - faz o "modelo completo (C/W/N-M)" aproximar-se da condição de "solo ideal" com o aumento da condutividade devido à dependência deste parâmetro com a frequência. Portanto a condição de solo ideal apresenta-se como uma boa aproximação para o cálculo da capacitância transversal.

Pelos resultados obtidos verifica-se que a correta representação do solo é importante para o cálculo da impedância longitudinal, mas não tem grande influência no cálculo dos parâmetros transversais. Pode-se, portanto, calcular a admitância transversal pelo método tradicional de se considerar a condição de solo ideal, ou seja, considerar o solo como plano condutor perfeito (condutividade infinita). Nota-se que a diferença entre o "modelo C/W/N-M" e o modelo de solo ideal não ultrapassa os $2 \%$ quando o solo é representado corretamente, mesmo para solos com alta resistividade.

\section{AGRADECIMENTOS}

Os autores agradecem o apoio financeiro das agências: FAPESP - Fundo de Apoio à Pesquisa no Estado de São Paulo; $\mathrm{CNPq}$ - Conselho Nacional de Desenvolvimento Científico e Tecnológico e CAPES - Coordenação de Aperfeiçoamento de pessoal de Nível Superior.

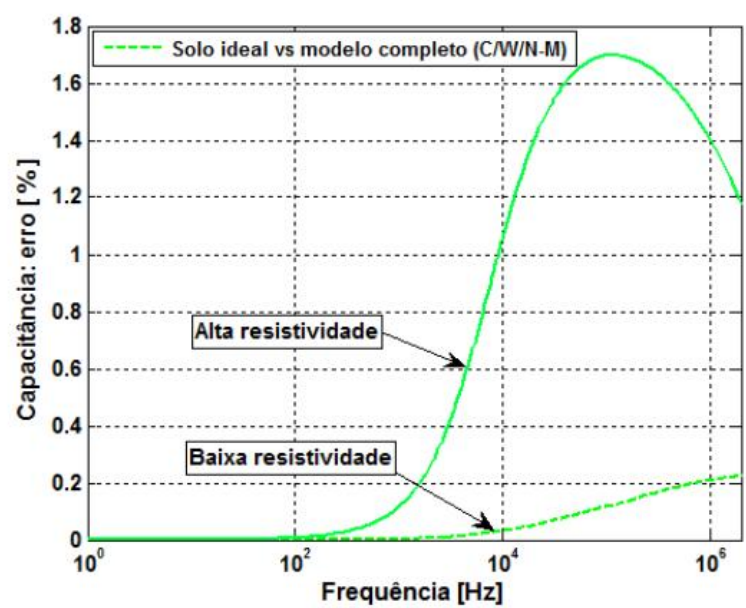

Figura 27: Capacitância por unidade de comprimento: Erro percentual entre modelo tradicional de cálculo e "modelo completo" com dependência dos parâmetros do solo com a frequência.

\section{REFERÊNCIAS BIBLIOGRÁFICAS}

Arismunandar, A. (1963), Capacitive Correction Factors for Transmission Lines to Include Finite Conductivity and Dielectric of the Earth, Proceedings of the AIEE Summer General Meeting and Nuclear Radiation Effects Conference, Toronto, Ont., Canadá, June 16-21, 1963, pp.: 436-455.

Carson, J. R. (1926), Wave Propagation in Overhead Wires, Bell System Technical Journal, vol. 5, pp.: 539554, 1926.

Deri, A.; Tevan, G.; Semlyen, A.; Castanheira, A. (1981), The Complex Ground Return Plane, a Simplified Model for Homogeneous and Multi-layer Earth Return, IEEE Trans. PAS, vol. 100, no. 8, pp.: 36863693, 1981.

Dwight, H. B. (1918), Skin Effect in Tubular and Flat Conductors, AIEE Transactions, Vol.37, pt. II, p.139-1403, 1918.

Nakagawa, M. (1981), Further Studies on Wave Propagation Along Overhead Transmission Lines: Effects of Admittance Correction, IEEE PES Winter Meeting, Atlanta, Georgia, 1981.

Portela, C. (1997), Frequency and Transient Behavior of Grounding Systems I- Physical and Methodological Aspects, Proceedings 1997 International Symposium on Electromagnetic Compatibility, pp.: 380-384, August Agosto 1997, United StatesEUA. 


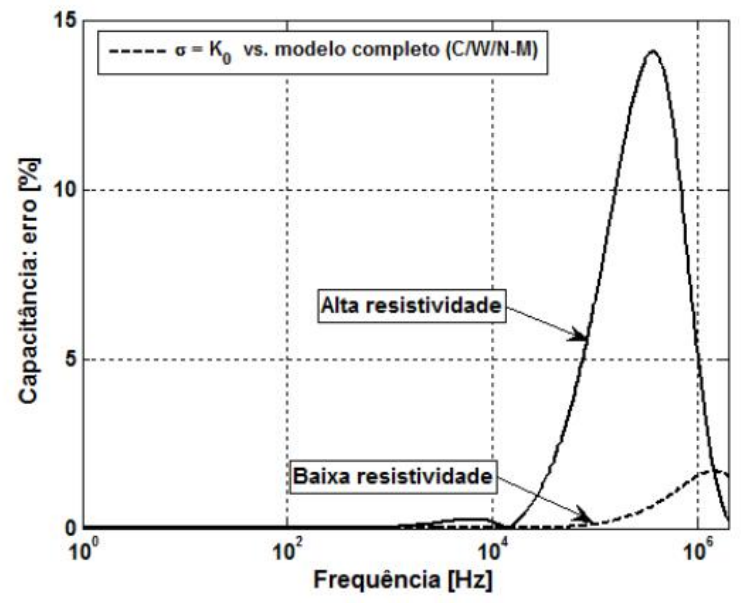

Figura 28: Capacitância por unidade de comprimento: Erro percentual entre modelo com condutividade constante e "modelo completo - C/W/N-M" com dependência dos parâmetros do solo com a frequência.

Portela, C. (1999), Measurement and Modeling of Soil Electromagnetic Behavior, Proceedings IEEE 1999 International Symposium on Electromagnetic Compatibility - EMC Society, pp.: 1004-1009, August 1999, United StatesEUA.

Portela, C. (2000), Grounding Requirement to Assure People and Equipment Safety Against Lightning, Proceedings IEEE 2000 International Symposium on Electromagnetic Compatibility, pp.: 969-974, August 2000, Washington DC, United StatesEUA.

Portela, C.; Gertrudes, J. B.; Tavares, M. C.; Pissolato, J. (2006), Earth Conductivity and Permittivity Data Measurements - Influence in Transmission Line Transient Performance. Electric Power Systems Research, v. 76, n. 2006, pp.: 907-915, 2006.

Portela, C.; Tavares, M.C.; Pissolato, J. (2003), Accurate Representation of Soil Behaviour for Transient Studies, IEE Proceedings on Genereation, Transmission and Distribution, Vol. 150/6, pp.: 734-744, Nov. 2003.

Rachidi, F.; Nucci, C. A.; Ianoz, M.; Mazzetti, C. (1996), Influence of a Lossy Ground on Lightning-induced Voltages on Overhead Lines, , IEEE Trans. Electromagn. Compatibility IEEE Transactions on EMC, vol.38, No. 2, Agosto, 1996.

Sunde, E. D. (1948), "Earth Conduction Effects in Transmission Systems", Princeton, New Jersey: Van Nostrand, 1948.

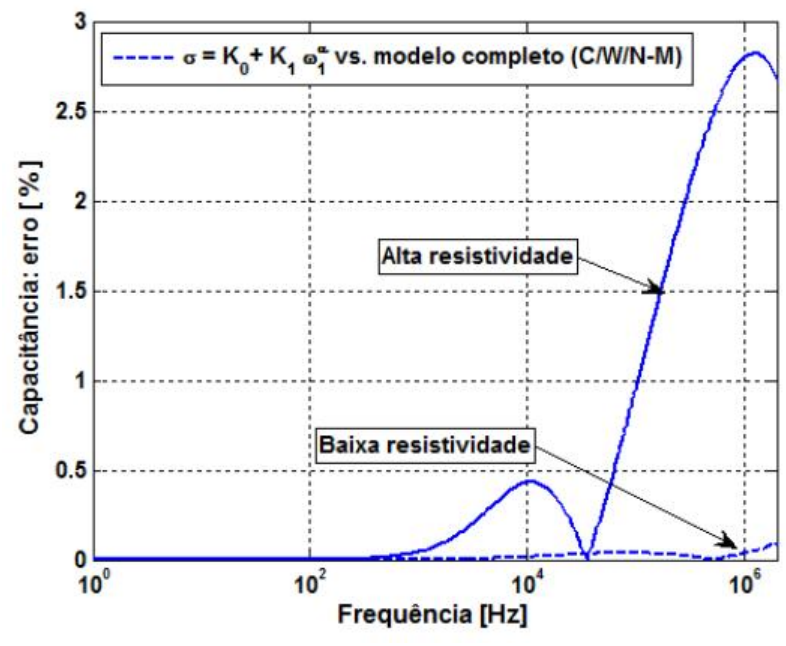

Figura 29: Capacitância por unidade de comprimento: Erro percentual entre modelo hipotético (onde somente $\sigma_{g}$ varia com a frequência) e "modelo completo" com dependência dos parâmetros do solo com a frequência.

Tesche, F. M. (1992), Comparison of the transmission line and scattering models for computing the HEMP response of overhead cables, IEEE Trans. Electromagn. Compatibility, vol. 34, May 1992.

Wise, W. H. (1931), Effect of Ground Permeability on Ground Return Circuits, Bell System Technical Journal, New York, vol. 10, Jul 1931, pp.: 472-484.

Wise, W. H. (1934), Propagation of High-Frequency Currents in Ground Return Circuits, Proceedings, Institute of Radio Engineers, New York, N.Y., vol. 22, Apr. 1934, pp.: 522-27. 